\title{
THE GROWTH OF DIMENSION OF COHOMOLOGY OF SEMIPOSITIVE LINE BUNDLES ON HERMITIAN MANIFOLDS
}

\author{
HUAN WANG
}

\begin{abstract}
In this paper, we study the dimension of cohomology of semipositive line bundles over Hermitian manifolds, and obtain an asymptotic estimate for the dimension of the space of harmonic $(0, q)$-forms with values in high tensor powers of a semipositive line bundle when the fundamental estimate holds. As applications, we estimate the dimension of cohomology of semipositive line bundles on $q$-convex manifolds, pseudo-convex domains, weakly 1-complete manifolds and complete manifolds. We also obtain the estimate of cohomology on compact manifolds with semipositive line bundles endowed with a Hermitian metric with analytic singularities and the related vanishing theorems.
\end{abstract}

\section{CONTENTS}

1. Introduction

2. Preliminaries

2.1. $L^{2}$-cohomology

2.2. $q$-convex complex manifolds and $\Gamma$-coverings

2.3. Kodaira Laplacian with $\bar{\partial}$-Neumann boundary conditions

2.4. Hermitian metric with analytic singularities on line bundles

3. Asymptotic estimate for $L^{2}$-cohomology with semipositive line bundles

3.1. q-convex manifolds

3.2. Pseudo-convex domains

3.3. Weakly 1-complete manifolds

3.4. Complete manifolds

3.5. Compact manifolds and coverings revisited

4. Analytic singular line bundles and vanishing theorems

4.1. Semipositive line bundles endowed metric with analytic singularities

4.2. Vanishing theorems for singular line bundles

Acknowledgements

References

\section{INTRODUCTION}

The purpose of this paper is to prove asymptotic estimates for the cohomology of semipositive line bundles over various non-compact manifolds. We generalize the asymptotics

Date: 23. Oct. 2018.

Key words and phrases. semipositive line bundles, cohomology, fundamental estimates, q-convex manifolds, pseudo-convex domains, weakly 1-complete manifolds, complete manifolds, singular Hermitian metric. 
obtained by Berndtsson [Ber02] in the compact case, which in turn refine the holomorphic Morse inequalities of Demailly [Dem85].

Let $X$ be a compact complex manifold, let $L$ be a holomorphic line bundle and $E$ be a holomorphic vector bundle on $X$. The Dolbeault cohomology $H^{0, q}\left(X, L^{k} \otimes E\right)$ plays a fundamental role in algebraic and complex geometry and is linked to the structure of the manifold, cf. [Dem, Dem85, MM07]. If $L$ is a positive line bundle, $H^{0, q}\left(X, L^{k} \otimes E\right)=0$ for $q \geq 1$ and $k$ large enough, by the Kodaira-Serre vanishing theorem (see e.g. [MM07, Theorem 1.5.6]) and this can be used to prove that global holomorphic sections of $L^{k} \otimes E$ give a projective embedding of $X$ for large $k$ (Kodaira embedding theorem).

Assume now that $L$ is semipositive. The solution of the Grauert-Riemenschneider conjecture [GR70] by Siu [Siu84] and Demailly [Dem85] shows that $\operatorname{dim} H^{0, q}\left(X, L^{k} \otimes\right.$ $E)=o\left(k^{n}\right)$ as $k \rightarrow \infty$ for $q \geq 1$. This can be used to show that $X$ is a Moishezon manifold, if $\left(L, h^{L}\right)$ is moreover positive at least at one point. Berndtsson [Ber02] showed that we have actually $\operatorname{dim} H^{0, q}\left(X, L^{k} \otimes E\right)=O\left(k^{n-q}\right)$ as $k \rightarrow \infty$ for $q \geq 1$.

We will consider first a very general situation where we can prove the decay of the cohomology groups as above. Let $(X, \omega)$ be a Hermitian manifold of dimension $n$. Let $d v_{X}:=\omega^{n} / n$ ! be the volume form on $X$. Let $\left(L, h^{L}\right)$ and $\left(E, h^{E}\right)$ be holomorphic Hermitian line bundles on $X$, where $L$ is a line bundle. We denote by $\left(L_{0, q}^{2}\left(X, L^{k} \otimes E\right),\|\cdot\|\right)$ the space of square integrable $(0, q)$-forms with values in $L^{k} \otimes E$ with respect to the $L^{2}$ inner product induced by the above data. We denote by $\bar{\partial}_{k}^{E}$ the maximal extension of the Dolbeault operator on $L_{0, \bullet}^{2}\left(X, L^{k} \otimes E\right)$ and by $\bar{\partial}_{k}^{E *}$ its Hilbert space adjoint. Let $\mathscr{H}^{0, q}\left(X, L^{k} \otimes E\right)$ be the space of harmonic $(0, q)$-forms with values in $L^{k} \otimes E$ on $X$.

For a given $0 \leq q \leq n$, we say that the concentration condition holds in bidegree $(0, q)$ for harmonic forms with values in $L^{k} \otimes E$ for large $k$, if there exists a compact subset $K \subset X$ and $C_{0}>0$ such that for sufficiently large $k$, we have

$$
\|s\|^{2} \leq C_{0} \int_{K}|s|^{2} d v_{X}
$$

for $s \in \operatorname{Ker}\left(\bar{\partial}_{k}^{E}\right) \cap \operatorname{Ker}\left(\bar{\partial}_{k}^{E *}\right) \cap L_{0, q}^{2}\left(X, L^{k} \otimes E\right)$. The set $K$ is called the exceptional compact set of the concentration. We say that the fundamental estimate holds in bidegree $(0, q)$ for forms with values in $L^{k} \otimes E$ for large $k$, if there exists a compact subset $K \subset X$ and $C_{0}>0$ such that for sufficiently large $k$, we have

$$
\|s\|^{2} \leq C_{0}\left(\left\|\bar{\partial}_{k}^{E} s\right\|^{2}+\left\|\bar{\partial}_{k}^{E, *} s\right\|^{2}+\int_{K}|s|^{2} d v_{X}\right),
$$

for $s \in \operatorname{Dom}\left(\bar{\partial}_{k}^{E}\right) \cap \operatorname{Dom}\left(\bar{\partial}_{k}^{E *}\right) \cap L_{0, q}^{2}\left(X, L^{k} \otimes E\right)$. The set $K$ is called the exceptional compact set of the estimate.

The first main result of this paper is an asymptotic estimate for $L^{2}$-cohomology with semipositive line bundles over Hermitian manifolds.

Theorem 1.1. Let $(X, \omega)$ be a Hermitian manifold of dimension $n$. Let $\left(L, h^{L}\right)$ and $\left(E, h^{E}\right)$ be holomorphic Hermitian line bundles on $X$. Assume that for some $1 \leq q \leq n$ the concentration condition holds in bidegree $(0, q)$ for harmonic forms with values in $L^{k} \otimes E$ for large $k$. Assume that $\left(L, h^{L}\right)$ is semipositive on a neighbourhood of the exceptional compact set $K$. Then there exists $C>0$ such that for sufficiently large $k$, we have

$$
\operatorname{dim} \mathscr{H}^{0, q}\left(X, L^{k} \otimes E\right) \leq C k^{n-q} .
$$


The same estimate also holds for reduced $L^{2}$-Dolbeault cohomology groups,

$$
\operatorname{dim} \bar{H}_{(2)}^{0, q}\left(X, L^{k} \otimes E\right) \leq C k^{n-q} .
$$

In particular, if the fundamental estimate holds in bidegree $(0, q)$ for forms with values in $L^{k} \otimes E$ for large $k$, the same estimate holds for $L^{2}$-Dolbeault cohomology groups,

$$
\operatorname{dim} H_{(2)}^{0, q}\left(X, L^{k} \otimes E\right) \leq C k^{n-q} .
$$

Note that holomorphic Morse inequalities for the $L^{2}$-cohomology were obtained under the assumption that the fundamental estimate holds in [MM07, Theorem 3.2.13]. They can only deliver an estimate $\operatorname{dim} H_{(2)}^{0, q}\left(X, L^{k} \otimes E\right)=o\left(k^{n}\right)$ as $k \rightarrow \infty$.

A geometric situation when Theorem 1.1 can be applied is the case of a semipositive line bundle on a complete Kähler manifold which polarizes the Kähler metric at infinity.

Theorem 1.2. Let $(X, \omega)$ be a complete Hermitian manifold of dimension $n$. Let $\left(L, h^{L}\right)$ and $\left(E, h^{E}\right)$ be holomorphic Hermitian line bundles on $X$. Assume there exists a compact subset $K \subset X$ such that $\sqrt{-1} R^{\left(L, h^{L}\right)}=\omega$ on $X \backslash K$ and $\left(L, h^{L}\right)$ is semipositive on $K$.

Then there exists $C>0$ such that for any $q \geq 1$ and sufficiently large $k$, we have

$$
\operatorname{dim} H_{(2)}^{0, q}\left(X, L^{k} \otimes E\right) \leq C k^{n-q} .
$$

Note that by [MM07, Theorem 3.3.5] we have

$$
\operatorname{dim} H_{(2)}^{0,0}\left(X, L^{k} \otimes K_{X}\right) \geq \frac{k^{n}}{n !} \int_{X} c_{1}\left(L, h^{L}\right)^{n}+o\left(k^{n}\right), \quad k \rightarrow \infty
$$

in the situation of Theorem 1.2. Moreover, the Bergman kernel of $H_{(2)}^{0,0}\left(X, L^{k} \otimes K_{X}\right)$ has an asymptotic expansion in powers of $k$ on the set where $c_{1}\left(L, h^{L}\right)>0$ by [HM14, Theorem 1.7].

We consider further the case of $q$-convex manifolds as application of Theorem 1.1. For general holomorphic Morse inequalities on $q$-convex manifolds see [MM07, Theorem 3.5.8].

Theorem 1.3. Let $X$ be a q-convex manifold of dimension $n$, and let $\left(L, h^{L}\right),\left(E, h^{E}\right)$ be holomorphic Hermitian line bundles on $X$. Let $\varrho$ be an exhaustion function of $X$ and $K$ the exceptional set. Let $\left(L, h^{L}\right)$ be semipositive on a sublevel set $X_{c}:=\{x \in X: \varrho(x)<c\}$ satisfying $K \subset X_{c}$, and let $\left(L, h^{L}\right)$ be positive on $X_{c} \backslash K$.

Then there exists $C>0$ such that for any $j \geq q$ and $k \geq 1$, we have

$$
\operatorname{dim} H^{j}\left(X, L^{k} \otimes E\right) \leq C k^{n-j} .
$$

We denote here by $H^{j}\left(X, L^{k} \otimes E\right)$ the cohomology groups of the sheaves $\mathcal{O}_{X}\left(L^{k} \otimes E\right)$ of holomoprhic sections of $L^{k} \otimes E$. They are isomorphic to the Dolbeault cohomology groups $H^{0, j}\left(X, L^{k} \otimes E\right)$.

In the case of 1-convex manifolds estimate (1.7) holds without additional hypothesis.

Theorem 1.4. Let $X$ be a 1-convex manifold of dimension $n$, and let $\left(L, h^{L}\right)$ and $\left(E, h^{E}\right)$ be holomorphic Hermitian line bundles on $X$. Let $\varrho$ be an exhaustion function of $X$ and $K$ the exceptional set. Let $\left(L, h^{L}\right)$ be semipositive on a sublevel set $X_{c}:=\{x \in X: \varrho(x)<c\}$ satisfying $K \subset X_{c}$. Then there exists $C>0$ such that for any $j \geq 1$ and $k \geq 1$ the estimate (1.7) holds. 
Similarly, we also have the estimates of cohomology over pseudoconvex domains and weakly 1-complete manifolds.

Theorem 1.5. Let $M \Subset X$ be a smooth (weakly) pseudoconvex domain in a complex manifold $X$ of dimension $n$. Let $\left(L, h^{L}\right)$ and $\left(E, h^{E}\right)$ be holomorphic Hermitian line bundles on $X$. Let $\left(L, h^{L}\right)$ be semipositive on $M$. Moreover, assume $\left(L, h^{L}\right)$ is positive in a neighbourhood of $b M$. Then there exists $C>0$ such that for any $q \geq 1$ and sufficiently large $k$, we have

$$
\operatorname{dim} H_{(2)}^{0, q}\left(X, L^{k} \otimes E\right) \leq C k^{n-q} .
$$

Theorem 1.6. Let $X$ be a weakly 1-complete manifold of dimension $n$. Let $\left(L, h^{L}\right)$ and $\left(E, h^{E}\right)$ be holomorphic Hermitian line bundles on $X$. Let $\left(L, h^{L}\right)$ be semipositive on $X$. Moreover, assume $\left(L, h^{L}\right)$ is positive on $X \backslash K$ for a compact subset $K \subset X$. Then there exists $C>0$ such that for any $q \geq 1$ and sufficiently large $k$, we have

$$
\operatorname{dim} H^{q}\left(X, L^{k} \otimes E\right) \leq C k^{n-q} .
$$

The next result is another generalization of [Ber02] for line bundles endowed with a Hermitian metric with analytic singularities. Let us recall that the analogue of the Kodaira vanishing theorem in the case of singular metrics is the Nadel vanishing theorem [Dem92, Nad90]. If $X$ is a compact Kähler manifold, $L$ and $E$ are holomorphic vector bundles with $\operatorname{rank}(L)=1$, and $h^{L}$ is a singular Hermitian metric such that $c_{1}\left(L, h^{L}\right)$ is a Kähler current, then $H^{q}\left(X, E \otimes L^{k} \otimes \mathscr{J}\left(h^{L^{k}}\right)\right)=0$ for $q \geq 1$ and $k$ suffiently large, where $\mathscr{J}\left(h^{L^{k}}\right)$ is the Nadel multiplier ideal sheaf associated to $h^{L^{k}}$. Bonavero [Bon98] obtained holomorphic Morse inequalities for singular Hermitian line bundles. These inequalities imply that for any $q \geq 1$ we have

$$
\operatorname{dim} H^{q}\left(X, L^{k} \otimes E \otimes \mathscr{J}\left(h^{L^{k}}\right)\right)=o\left(k^{n}\right), \quad k \rightarrow \infty,
$$

if the curvature $c_{1}(L, h)$ is semipositive on the set of points where it is smooth. We obtain the following refinement of (1.10).

Theorem 1.7. Let $X$ be a compact complex manifold of dimension $n$ and let $L$ be a holomorphic line bundle on $X$ endowed with a Hermitian metric $h^{L}$ with analytic singularities. Let $\left(E, h^{E}\right)$ be a holomorphic Hermitian line bundle on $X$. Assume $c_{1}\left(L, h^{L}\right) \geq 0$ on the set $\left\{x \in X: h^{L}\right.$ smooth on a neighborhood of $\left.x\right\}$.

Then there exists $C>0$ such that for any $q \geq 1$ and $k \geq 1$, we have

$$
\operatorname{dim} H^{q}\left(X, L^{k} \otimes E \otimes \mathscr{J}\left(h^{L^{k}}\right)\right) \leq C k^{n-q} .
$$

In particular, this estimate still holds when $c_{1}\left(L, h^{L}\right)$ is a positive current on $X$.

Remark 1.8. Under the hypothesis of Theorem 1.7, if $c_{1}\left(L, h^{L}\right) \geq 0$ on the set $\{x \in X$ : $h^{L}$ smooth on a neighborhood of $\left.x\right\}$ and positive at least at one point, then there exist $C_{1}>0$ and $C_{2}>0$ such that for sufficiently large $k$, we have

$$
C_{1} k^{n} \leq \operatorname{dim} H^{0}\left(X, L^{k} \otimes E \otimes \mathscr{J}\left(h^{L^{k}}\right)\right) \leq C_{2} k^{n} .
$$

Thus $L$ is big and $X$ is Moishezon. 
The last results are analytic singular versions of the vanishing theorem for partially positive line bundles [Dem, Ch.VII. (5.1) Theorem] and Siu's vanishing theorem [Siu85, Theorem 1 (Page 175)] ([Rie71, Appendix, Theorem 6 and Theorem 3 (ii)] implies that, for a compact complex manifold $X$ and a holomorphic Hermitian line bundle $\left(L, h^{L}\right)$ on $X$, if $X$ is Moishezon and $c_{1}\left(L, h^{L}\right) \geq 0$ on $X$ and $c_{1}\left(L, h^{L}\right)>0$ at least at one point, then $H^{q}\left(X, K_{X} \otimes L\right)=0$ for all $q \geq 1$. Furthermore, Siu's resolution [Siu85, Theorem 1 (Page 175)] of Grauert-Riemenschneider conjecture shows that the Moishezon assumption is unnecessary, and we called it Siu's vanishing theorem, see also [Dem, Ch.VII. (3.5) Theorem]).

Theorem 1.9. Let $X$ be a compact manifold of dimension $n$, let $L$ and $E$ be holomorphic vector bundles with $\operatorname{rank}(L)=1$. Let $h^{L}$ be the Hermitian metric on $L$ with analytic singularities. Assume $c_{1}\left(L, h^{L}\right)$ has at least $n-s+1$ positive eigenvalues on the set $\{x \in$ $X: h^{L}$ smooth on a neighborhood of $\left.x\right\}$.

Then, for any $q \geq s$ and sufficiently large $k$, we have

$$
H^{q}\left(X, E \otimes L^{k} \otimes \mathscr{J}\left(h^{L^{k}}\right)\right)=0 .
$$

Therefore, it follows that

$$
\sum_{q=0}^{s-1}(-1)^{q} \operatorname{dim} H^{q}\left(X, E \otimes L^{k} \otimes \mathscr{J}\left(h^{L^{k}}\right)\right)=\frac{k^{n}}{n !} \int_{X(\leq s-1)} c_{1}\left(L, h^{L}\right)^{n}+o\left(k^{n}\right) .
$$

Note that for a Hermitian metric with analytic singularities on line bundles over connected compact complex manifolds (see Definition 2.9), the non-negative rational number $c$ does not depend on the local form (2.9) of the weight $\varphi$.

Theorem 1.10. Let $X$ be a compact manifold of dimension $n$, let $L$ be a holomorphic line bundle. Let $h^{L}$ be the Hermitian metric on $L$ with analytic singularities. Assume $c_{1}\left(L, h^{L}\right) \geq$ 0 on the set $\left\{x \in X: h^{L}\right.$ smooth on a neighborhood of $\left.x\right\}$ and $c_{1}\left(L, h^{L}\right)>0$ at least at one point.

Then, for any $q \geq 1, k \geq 1$ and $m \in \mathbb{N} \backslash\{0\}$ satisfying $m c \in \mathbb{N}$ with non-negative rational number $c$ in (2.9), we have

$$
H^{q}\left(X, K_{X} \otimes L^{k m} \otimes \mathscr{J}\left(h^{L^{k m}}\right)\right)=0 .
$$

In this paper we consider the cohomology spaces on general (possibly non-compact) complex manifolds with semipositive line bundles. With the fundamental estimates fulfilled, Theorem 1.1 give an estimate of $L^{2}$-Dolbeaut cohomology on arbitrary complex manifolds. On one hand, it generalizes [Ber02] to general complex manifolds in the context of $L^{2}$-cohomology; on the other hand, it leads to the refinement of the estimates for $q$-convex manifolds, pseudoconvex domains, weakly 1-convex manifolds, complete manifolds, and semipositive line bundle endowed metric with analytic singularities, see Theorem 1.3, 1.5, 1.6, 1.2, and 1.7, respectively. Note also that the magnitude $k^{n-q}$ in Theorem 1.1, Theorem 1.3, Theorem 1.6, Theorem 1.2, Theorem 1.7 and Theorem 3.22 cannot be improved in general, see [Ber02, Proposition 4.2].

Our paper is organized in the following way. In Section 2 we introduce the notations and recall the necessary facts. In Section 3, we give an asymptotic estimate for $\mathrm{L}^{2}$ cohomology with semipositive line bundles on Hermitian manifolds, which is a uniform 
approach to consider semipositive line bundles on complex manifolds. As applications, we obtain the estimate of growth of dimension in certain possibly non-compact complex manifolds. In additional, we revisited the compact and covering manifolds in this context. In Section 4 we prove the estimate of cohomology still holds when the Hermitian metric of the line bundle has analytic singularities. Meanwhile, two vanishing theorems of line bundles are given in the setting of analytic singular Hermitian metric. The techniques and formulations are mainly based on Berndtsson [Ber02], Ma-Marinescu [MM07] and [Wan16].

\section{PRELIMINARIES}

2.1. $L^{2}$-cohomology. Let $(X, \omega)$ be a Hermitian manifold of dimension $n$ and $\left(F, h^{F}\right)$ be a holomorphic Hermitian vector bundle over $X$. Let $\Omega^{p, q}(X, F)$ be the space of smooth $(p, q)$-forms on $X$ with values in $F$ for $p, q \in \mathbb{N}$. If the $\operatorname{rank}(F)=1$, the curvature of $\left(F, h^{F}\right)$ is defined by $R^{F}=\bar{\partial} \partial \log |s|_{h^{F}}^{2}$ for any local holomorphic frame $s$, then the Chern-Weil form of the first Chern class of $F$ is $c_{1}\left(F, h^{F}\right)=\frac{\sqrt{-1}}{2 \pi} R^{F}$, which is a real $(1,1)$ form on $X$. The volume form is given by $d v_{X}:=\omega_{n}:=\frac{\omega^{n}}{n !}$. We use the notion of positive $(p, p)$-form given by [Dem, Chapter III, $\S 1,(1.1)(1.2)(1.5)(1.7)]$. If a $(p, p)$-form $T$ is positive, we write $T \geq 0$.

Definition 2.1. We say a holomorphic Hermitian line bundle $\left(L, h^{L}\right)$ is semipositive on $X$, if $c_{1}\left(L, h^{L}\right)$ is positive semi-definite on $X$, equivalently $c_{1}\left(L, h^{L}\right) \geq 0$. For simplifying notations, we also denote $L \geq 0$.

Let $\Omega_{0}^{p, q}(X, F)$ be the subspace of $\Omega^{p, q}(X, F)$ consisting of elements with compact support. The $L^{2}$-scalar product on $\Omega_{0}^{p, q}(X, F)$ given by

$$
\left\langle s_{1}, s_{2}\right\rangle=\int_{X}\left\langle s_{1}(x), s_{2}(x)\right\rangle_{h} d v_{X}(x)
$$

where $\langle\cdot, \cdot\rangle_{h}:=\langle\cdot, \cdot\rangle_{h^{F}, \omega}$ is the pointwise Hermitian inner product induced by $\omega$ and $h^{F}$. We denote by $L_{p, q}^{2}(X, F)$, the $L^{2}$ completion of $\Omega_{0}^{p, q}(X, F)$.

Let $\bar{\partial}^{F}: \Omega_{0}^{p, q}(X, F) \rightarrow L_{p, q+1}^{2}(X, F)$ be the Dolbeault operator and let $\bar{\partial}_{\max }^{F}$ be its maximal extension (see [MM07, Lemma 3.1.1]). From now on we still denote the maximal extension by $\bar{\partial}^{F}:=\bar{\partial}_{\max }^{F}$ and the associated Hilbert space adjoint by $\bar{\partial}^{F *}:=\bar{\partial}_{H}^{F *}:=\left(\bar{\partial}_{\max }^{F}\right)_{H}^{*}$ for simplifying the notations. Consider the complex of closed, densely defined operators $L_{p, q-1}^{2}(X, F) \stackrel{\bar{\partial}^{F}}{\longrightarrow} L_{p, q}^{2}(X, F) \stackrel{\bar{\partial}^{F}}{\longrightarrow} L_{p, q+1}^{2}(X, F)$, then $\left(\bar{\partial}^{F}\right)^{2}=0$. By [MM07, Proposition 3.1.2], the operator defined by

$$
\begin{aligned}
\operatorname{Dom}\left(\square^{F}\right) & =\left\{s \in \operatorname{Dom}\left(\bar{\partial}^{F}\right) \cap \operatorname{Dom}\left(\bar{\partial}^{F *}\right): \bar{\partial}^{F} s \in \operatorname{Dom}\left(\bar{\partial}^{F *}\right), \bar{\partial}^{F *} s \in \operatorname{Dom}\left(\bar{\partial}^{F}\right)\right\}, \\
\square^{F} s & =\bar{\partial}^{F} \bar{\partial}^{F *} s+\bar{\partial}^{F *} \bar{\partial}^{F} s \quad \text { for } s \in \operatorname{Dom}\left(\square^{F}\right),
\end{aligned}
$$

is a positive, self-adjoint extension of Kodaira Laplacian, called the Gaffney extension.

Definition 2.2. The space of harmonic forms $\mathscr{H}^{p, q}(X, F)$ is defined by

$$
\mathscr{H}^{p, q}(X, F):=\operatorname{Ker}\left(\square^{F}\right)=\left\{s \in \operatorname{Dom}\left(\square^{F}\right) \cap L_{p, q}^{2}(X, F): \square^{F} s=0\right\} .
$$


The $q$-th reduced $L^{2}$-Dolbeault cohomology is defined by

$$
\bar{H}_{(2)}^{0, q}(X, F):=\frac{\operatorname{Ker}\left(\bar{\partial}^{F}\right) \cap L_{0, q}^{2}(X, F)}{\left[\operatorname{Im}\left(\bar{\partial}^{F}\right) \cap L_{0, q}^{2}(X, F)\right]},
$$

where $[V]$ denotes the closure of the space $V$. The $q$-th (non-reduced) $L^{2}$-Dolbeault cohomology is defined by

$$
H_{(2)}^{0, q}(X, F):=\frac{\operatorname{Ker}\left(\bar{\partial}^{F}\right) \cap L_{0, q}^{2}(X, F)}{\operatorname{Im}\left(\bar{\partial}^{F}\right) \cap L_{0, q}^{2}(X, F)} .
$$

According to the general regularity theorem of elliptic operators (cf. [MM07, Theorem A.3.4]), $s \in \mathscr{H}^{p, q}(X, F)$ implies $s \in \Omega^{p, q}(X, F)$. By weak Hodge decomposition (cf. [MM07, (3.1.21) (3.1.22)]), we have a canonical isomorphism

$$
\bar{H}_{(2)}^{0, q}(X, F) \cong \mathscr{H}^{0, q}(X, F)
$$

for any $q \in \mathbb{N}$, which associates to each cohomology class its unique harmonic representative. The $q$-th cohomology of the sheaf of holomorphic sections of $F$ is isomorphic to the the $q$-th Dolbeault cohomology, $H^{q}(X, F) \cong H^{0, q}(X, F)$.

For a given $0 \leq q \leq n$, we say the fundamental estimate holds in bidegree $(0, q)$ for forms with values in $F$, if there exists a compact subset $K \subset X$ and $C>0$ such that

$$
\|s\|^{2} \leq C\left(\left\|\bar{\partial}^{F} s\right\|^{2}+\left\|\bar{\partial}^{F *}\right\|^{2}+\int_{K}|s|^{2} d v_{X}\right)
$$

for $s \in \operatorname{Dom}\left(\bar{\partial}^{F}\right) \cap \operatorname{Dom}\left(\bar{\partial}^{F, *}\right) \cap L_{0, q}^{2}(X, F) . K$ is called the exceptional compact set of the estimate. If the fundamental estimate holds in bidegree $(0, q)$ for forms with values in $F$, the reduced and non-reduced $L^{2}$-Dolbeault cohomology coincide, see [MM07, Theorem 3.1.8].

For a given $0 \leq q \leq n$, we say that the concentration condition holds in bidegree $(0, q)$ for harmonic forms with values in $F$, if there exists a compact subset $K \subset X$ and $C>0$ such that

$$
\|s\|^{2} \leq C \int_{K}|s|^{2} d v_{X}
$$

for $s \in \operatorname{Ker}\left(\bar{\partial}^{F}\right) \cap \operatorname{Ker}\left(\bar{\partial}^{F *}\right) \cap L_{0, q}^{2}(X, F)$. The compact set $K$ is called the exceptional compact set of the concentration. Note if the fundamental estimate holds in bidegree $(0, q)$ for forms with values in $F$, the concentration condition holds in bidegree $(0, q)$ for harmonic forms with values in $F$.

\section{2. q-convex complex manifolds and $\Gamma$-coverings.}

Definition 2.3 ([AG62]). A complex manifold $X$ of dimension $n$ is called $q$-convex if there exists a smooth function $\varrho \in \mathscr{C}^{\infty}(X, \mathbb{R})$ such that the sublevel set $X_{c}=\{\varrho<c\} \Subset X$ for all $c \in \mathbb{R}$ and the complex Hessian $\partial \bar{\partial} \varrho$ has $n-q+1$ positive eigenvalues outside a compact subset $K \subset X$. Here $X_{c} \Subset X$ means that the closure $\bar{X}_{c}$ is compact in $X$. We call $\varrho$ an exhaustion function and $K$ exceptional set. We say $X$ is $q$-complete if $K=\emptyset$ in additional. 
Definition 2.4. A complex manifold $X$ of dimension $n$ is called a $q$-convex manifold with a plurisubharmonic exhaustion function near the exceptional set, if there exists a compact subset $K \subset X$ and a smooth function $\varrho \in \mathscr{C}^{\infty}(X, \mathbb{R})$ such that the sublevel set $X_{c}:=\{\varrho<c\} \Subset X$ for all $c \in \mathbb{R}$, and $\sqrt{-1} \partial \bar{\partial} \varrho$ has at least $n-q+1$ positive eigenvalues on $X \backslash K$ and $\sqrt{-1} \partial \bar{\partial} \varrho \geq 0$ on $X_{c} \backslash K$ for some $X_{c}$ with $K \subset X_{c}$.

Let $M$ be a relatively compact domain with smooth boundary $b M$ in a complex manifold $X$. Let $\rho \in \mathscr{C}^{\infty}(X, \mathbb{R})$ such that $M=\{x \in X: \rho(x)<0\}$ and $d \rho \neq 0$ on $b M=\{x \in X: \rho(x)=0\}$. We denote the closure of $M$ by $\bar{M}=M \cup b M$. We say that $\rho$ is a defining function of $M$. Let $T^{(1,0)} b M:=\left\{v \in T_{x}^{(1,0)} X: \partial \varrho(v)=0\right\}$ be the analytic tangent bundle to $b M$ at $x \in b M$. The Levi form of $\rho$ is the 2-form $\mathscr{L}_{\rho}:=\partial \bar{\partial} \rho \in \mathscr{C}^{\infty}\left(b M, T^{(1,0) *} b M \otimes T^{(0,1) *} b M\right)$.

Definition 2.5. A relatively compact domain $M$ with smooth boundary $b M$ in a complex manifold $X$ is called strongly (resp. (weakly)) pseudoconvex if the Levi form $\mathscr{L}_{\rho}$ is positive definite (resp. semidefinite).

Note that any strongly pseudoconvex domain is 1-convex.

Definition 2.6. A complex manifold $X$ is called weakly 1-complete if there exists a smooth plurisubharmonic function $\varphi \in \mathscr{C}^{\infty}(X, \mathbb{R})$ such that $\{x \in X: \varphi(x)<c\} \Subset X$ for any $c \in \mathbb{R} . \varphi$ is called an exhaustion function.

Note that any 1-convex manifold is weakly 1-complete.

Definition 2.7. A Hermitian manifold $(X, \omega)$ is called complete, if all geodesics are defined for all time for the underlying Riemannian manifold.

If $(X, \omega)$ is complete, for arbitrary holomorphic Hermitian vector bundle $\left(F, h^{F}\right)$ on $X$, $\Omega_{0}^{0, \bullet}(X, F)$ is dense in $\operatorname{Dom}\left(\bar{\partial}^{F}\right), \operatorname{Dom}\left(\bar{\partial}_{H}^{F *}\right)$ and $\operatorname{Dom}\left(\bar{\partial}^{F}\right) \cap \operatorname{Dom}\left(\bar{\partial}_{H}^{F *}\right)$ in the graph-norms of $\bar{\partial}^{F}, \bar{\partial}_{H}^{F *}$ and $\bar{\partial}^{E}+\bar{\partial}_{H}^{E *}$ respectively, see [MM07, Lemma 3.3.1 (Andreotti-Vesentini), Corollary 3.3.3]. Here the graph-norm is defined by $\|s\|+\|R s\|$ for $s \in \operatorname{Dom}(R)$.

Definition 2.8. Let $(X, \omega)$ be a Hermitian manifold of dimension $n$ on which a discrete group $\Gamma$ acts holomorphically, freely and properly such that $\omega$ is a $\Gamma$-invariant Hermitian metric and the quotient $X / \Gamma$ is compact. We say $X$ is a $\Gamma$-covering manifold.

2.3. Kodaira Laplacian with $\bar{\partial}$-Neumann boundary conditions. Let $(X, \omega)$ be a Hermitian manifold of dimension $n$ and $\left(F, h^{F}\right)$ be a holomorphic Hermitian vector bundles over $X$. Let $M$ be a relatively compact domain in $X$. Let $\rho$ be a defining function of $M$ satisfying $M=\{x \in X: \rho(x)<0\}$ and $|d \rho|=1$ on $b M$, where the pointwise norm $|\cdot|$ is given by $g^{T X}$ associated to $\omega$.

Let $e_{n} \in T X$ be the inward pointing unit normal at $b M$ and $e_{n}^{(0,1)}$ its projection on $T^{(0,1)} X$. In a local orthonormal frame $\left\{w_{1}, \cdots, \omega_{n}\right\}$ of $T^{(1,0)} X$, we have $e_{n}^{(0,1)}=$ $-\sum_{j=1}^{n} w_{j}(\rho) \bar{w}_{j}$. Let $B^{0, q}(X, F):=\left\{s \in \Omega^{0, q}(\bar{M}, F): i_{e_{n}^{(0,1)}} s=0\right.$ on $\left.b M\right\}$. We have $B^{0, q}(M, F)=\operatorname{Dom}\left(\bar{\partial}_{H}^{F *}\right) \cap \Omega^{0, q}(\bar{M}, F)$ and the Hilbert space adjoint $\bar{\partial}_{H}^{F *}$ of $\bar{\partial}^{F}$ coincides with the formal adjoint $\bar{\partial}^{F *}$ of $\bar{\partial}^{F}$ on $B^{0, q}(M, F)$, see [MM07, Proposition 1.4.19]. We consider the operator $\square_{N} s=\bar{\partial}^{F} \bar{\partial}^{F *} s+\bar{\partial}^{F *} \bar{\partial}^{F} s$ for $s \in \operatorname{Dom}\left(\square_{N}\right):=\left\{s \in B^{0, q}(M, F)\right.$ : $\left.\bar{\partial}^{F} s \in B^{0, q+1}(M, F)\right\}$. The Friedrichs extension of $\square_{N}$ is a self-adjoint operator and 
is called the Kodaira Laplacian with $\bar{\partial}$-Neumann boundary conditions, which coincides with the Gaffney extension of the Kodaira Laplacian, see [MM07, Proposition 3.5.2]. Note $\Omega^{0, \bullet}(\bar{M}, F)$ is dense in $\operatorname{Dom}\left(\bar{\partial}^{F}\right)$ in the graph-norms of $\bar{\partial}^{F}$, and $B^{0, \bullet}(M, F)$ is dense in $\operatorname{Dom}\left(\bar{\partial}_{H}^{F *}\right)$ and in $\operatorname{Dom}\left(\bar{\partial}^{F}\right) \cap \operatorname{Dom}\left(\bar{\partial}_{H}^{F *}\right)$ in the graph-norms of $\bar{\partial}_{H}^{F *}$ and $\bar{\partial}^{E}+\bar{\partial}_{H}^{E *}$, respectively, see [MM07, Lemma 3.5.1]. Here the graph-norm is defined by $\|s\|+\|R s\|$ for $s \in \operatorname{Dom}(R)$.

\subsection{Hermitian metric with analytic singularities on line bundles.}

Definition 2.9. Let $X$ be a connected compact complex manifold of dimension $n$ and $L$ a holomorphic line bundle on $X$. On $L$ we say $h^{L}$ is a Hermitian metric with analytic singularities, if there exists a smooth hermitian metric $h_{0}^{L}$ and a function $\varphi \in L_{l o c}^{1}(X, \mathbb{R})$ with locally

$$
\varphi=\frac{c}{2} \log \left(\sum_{j \in J}\left|f_{j}\right|^{2}\right)+\psi,
$$

where $J$ is at most countable, $c$ is a non-negative rational number, $f_{j}$ are non-zero holomorphic functions and $\psi$ is a smooth function, such that $h^{L}=h_{0}^{L} e^{-2 \varphi}$.

For any local holomorphic frame $e_{L}$ of $L, h_{0}^{L}\left(e_{L}, e_{L}\right)=e^{-2 \psi_{0}}$, where $\psi_{0}$ is smooth, thus the local weights of $h^{L}$ is given by $\frac{c}{2} \log \left(\sum_{J}\left|f_{j}\right|^{2}\right)+\left(\psi+\psi_{0}\right)$. Because locally $\psi+\psi_{0}$ is smooth, we use (2.9) to represent the local weight of $h^{L}$ for simplifying notations.

For $\varphi \in L_{l o c}^{1}(X, \mathbb{R})$, the Nadel multiplier ideal sheaf $\mathscr{J}(\varphi)$ is the ideal subsheaf of germs of holomorphic functions $f \in \mathscr{O}_{X, x}$ such that $|f|^{2} e^{-2 \varphi}$ is integrable with respect to the Lebesgue measure in local coordinates near $x$. We define $\mathscr{J}\left(h^{L}\right):=\mathscr{J}(\varphi)$, which does not depend on the choice of $\varphi$, see [Dem92] or [MM07, Definition 2.3.13].

\section{ASYMPTOTIC ESTIMATE FOR $L^{2}$-COHOMOLOGY WITH SEMIPOSITIVE LINE BUNDLES}

Let $(X, \omega)$ be a Hermitian manifold of dimension $n$ and let $\left(L, h^{L}\right)$ and $\left(E, h^{E}\right)$ be holomorphic Hermitian line bundles over $X$. Let $\mathscr{H}^{0, q}\left(X, L^{k} \otimes E\right)$ be the space of harmonic $(0, q)$-forms with values in $L^{k} \otimes E$. Let $\left\{s_{j}^{k}\right\}_{j \geq 1}$ be an orthonormal basis and denote by $B_{k}^{q}$ the Bergman density function defined by

$$
B_{k}^{q}(x)=\sum_{j \geq 1}\left|s_{j}^{k}(x)\right|_{h_{k}, \omega}^{2}, x \in X,
$$

where $|\cdot|_{h_{k}, \omega}$ is the pointwise norm of a form, see [Wan16]. The function [3.1) is welldefined by an adaptation of [CM15, Lemma 3.1] to form case. By replacing $E \otimes \Lambda^{n}\left(T^{(1,0)} X\right)$ for $E$ in $\mathscr{H}^{n, q}\left(X, L^{k} \otimes E\right)$, we can rephrase [Wan16, Theorem 1.1] as follows.

Theorem 3.1. Let $(X, \omega)$ be a Hermitian manifold of dimension $n$ and let $\left(L, h^{L}\right)$ and $\left(E, h^{E}\right)$ be holomorphic Hermitian line bundles over $X$. Let $K \subset X$ be a compact subset and assume that $\left(L, h^{L}\right)$ is semipositive on a neighborhood of $K$.

Then there exists $C>0$ depending on the compact set $K$, the metric $\omega$ and the bundles $\left(L, h^{L}\right)$ and $\left(E, h^{E}\right)$, such that for any $x \in K, k \geq 1$ and $q \geq 1$,

$$
B_{k}^{q}(x) \leq C k^{n-q},
$$

where $B_{k}^{q}(x)$ is the Bergman density function (3.1) of harmonic $(0, q)$-forms with values in $L^{k} \otimes E$. 
A general result on asymptotic estimate for $L^{2}$-cohomology with semipositive line bundles over Hermitian manifolds follows immediately.

Theorem 3.2 (Theorem 1.1). Let $(X, \omega)$ be a Hermitian manifold of dimension $n$. Let $\left(L, h^{L}\right)$ and $\left(E, h^{E}\right)$ be holomorphic Hermitian line bundles on $X$. Let $1 \leq q \leq n$. Assume the concentration condition holds in bidegree $(0, q)$ for harmonic forms with values in $L^{k} \otimes E$ for large $k$. Moreover, assume $\left(L, h^{L}\right)$ is semipositive on a neighbourhood of the exceptional set $K$. Then there exists $C>0$ such that for sufficiently large $k$ we have

$$
\operatorname{dim} \mathscr{H}^{0, q}\left(X, L^{k} \otimes E\right) \leq C k^{n-q} .
$$

The same estimate also holds for reduced $L^{2}$-Dolbeault cohomology groups,

$$
\operatorname{dim} \bar{H}_{(2)}^{0, q}\left(X, L^{k} \otimes E\right) \leq C k^{n-q} .
$$

In particular, if the fundamental estimate holds in bidegree $(0, q)$ for forms with values in $L^{k} \otimes E$ for large $k$, the same estimate holds for $L^{2}$-Dolbeault cohomology groups

$$
\operatorname{dim} H_{(2)}^{0, q}\left(X, L^{k} \otimes E\right) \leq C k^{n-q} .
$$

Proof. By Theorem 3.1 and the concentration condition, we have

$$
\begin{aligned}
\operatorname{dim} \bar{H}_{(2)}^{(0, q)} & =\operatorname{dim} \mathscr{H}^{0, q}\left(X, L^{k} \otimes E\right) \\
& =\sum_{j \geq 1}\left\|s_{j}^{k}\right\|^{2} \leq C_{0} \int_{K} B_{k}^{q}(x) d v_{X} \leq C_{0} C \operatorname{vol}(K) k^{n-q}
\end{aligned}
$$

for sufficiently large $k$. Note that $H_{(2)}^{0, q}(X, F)=\bar{H}_{(2)}^{0, q}(X, F)$ and the dimension is finite, when the fundamental estimate holds in bidegree $(0, q)$ for forms with values in a holomorphic Hermitian vector bundle $\left(F, h^{F}\right)$ by [MM07, Theorem 3.1.8].

\section{1. q-convex manifolds.}

3.1.1. Exhaustion functions with the plurisubharmonic near the exceptional set. In this section we prove the following general result about the growth of the cohomology of $q$-convex manifolds.

Theorem 3.3. Let $X$ be a q-convex manifold of dimension $n$, and let $\left(L, h^{L}\right),\left(E, h^{E}\right)$ be holomorphic Hermitian line bundles on $X$. Let $\varrho$ be an exhaustion function of $X$ and $K$ the exceptional set. Let $\left(L, h^{L}\right)$ be semipositive on a sublevel set $X_{c}:=\{x \in X: \varrho(x)<c\}$ satisfying $K \subset X_{c}$, and let $\sqrt{-1} \partial \bar{\partial} \varrho \geq 0$ on $X_{c} \backslash K$. Then there exists $C>0$ such that for any $j \geq q$ and $k \geq 1$, we have

$$
\operatorname{dim} H^{j}\left(X, L^{k} \otimes E\right) \leq C k^{n-j} .
$$

Let $X$ be a $q$-convex manifold of dimension $n$, let $\varrho$ be a plurisubharmonic exhaustion function of $X$ and $K$ the exceptional set. By the definition, $\varrho \in \mathscr{C}^{\infty}(X, \mathbb{R})$ satisfies $X_{c}:=\{\varrho<c\} \Subset X$ for all $c \in \mathbb{R}, \sqrt{-1} \partial \bar{\partial} \varrho$ has $n-q+1$ positive eigenvalues on $X \backslash K$. In this section, we fix real numbers $u_{0}, u$ and $v$ satisfying $u_{0}<u<c<v$ and $K \subset X_{u_{0}}$.

Let $\left(L, h^{L}\right),\left(E, h^{E}\right)$ be holomorphic Hermitian line bundles on $X$. We have that the fundamental estimate holds in bidegree $(0, j)$ for forms with values in $L^{k} \otimes E$ for large $k$ and each $q \leq j \leq n$ on $X_{c}$ when $X$ is a $q$-convex manifold, see Proposition 3.8, which 
was obtained in [MM07, Theorem 3.5.8] for the proof of Morse inequalities on $q$-convex manifold. For the sake of completeness, we prove it here.

Firstly, we choose now a Hermitian metric on a $q$-convex manifold $X$.

Lemma 3.4. ([MM07, Lemma 3.5.3]). For any $C_{1}>0$ there exists a metric $g^{T X}$ (with Hermitian form $\omega$ ) on $X$ such that for any $j \geq q$ and any holomorphic Hermitian vector bundle $\left(F, h^{F}\right)$ on $X$,

$$
\left\langle(\partial \bar{\partial} \varrho)\left(w_{l}, \bar{w}_{k}\right) \bar{w}^{k} \wedge i_{\bar{w}_{l}} s, s\right\rangle_{h} \geq C_{1}|s|^{2}, \quad s \in \Omega_{0}^{0, j}\left(X_{v} \backslash \bar{X}_{u}, F\right),
$$

where $\left\{w_{l}\right\}_{l=1}^{n}$ is a local orthonormal frame of $T^{(1,0)} X$ with dual frame $\left\{w^{l}\right\}_{l=1}^{n}$ of $T^{(1,0) *} X$.

Now we consider the $q$-convex manifold $X$ associated with the metric $\omega$ obtained above as a Hermitian manifold $(X, \omega)$. Note for arbitrary holomorphic vector bundle $F$ on a relatively compact domain $M$ in $X$, the Hilbert space adjoint $\bar{\partial}_{H}^{F *}$ of $\bar{\partial}^{F}$ coincides with the formal adjoint $\bar{\partial}^{F *}$ of $\bar{\partial}^{F}$ on $B^{0, j}(M, F)=\operatorname{Dom}\left(\bar{\partial}_{H}^{F *}\right) \cap \Omega^{0, j}(\bar{M}, F), 1 \leq j \leq n$. So we simply use the notion $\bar{\partial}^{F *}$ on $B^{0, j}(M, F), 1 \leq j \leq n$.

Secondly, we modify Hermitian metric $h_{\chi}^{\bar{L}}$ on $L$ and show the fundamental estimate fulfilled. Let $\chi(t) \in \mathscr{C}^{\infty}(\mathbb{R})$ such that $\chi^{\prime}(t) \geq 0, \chi^{\prime \prime}(t) \geq 0$. We define a Hermitian metric $h_{\chi}^{L^{k}}:=h^{L^{k}} e^{-k \chi(\varrho)}$ on $L^{k}$ for each $k \geq 1$ and set $L_{\chi}^{k}:=\left(L^{k}, h_{\chi}^{L^{k}}\right)$. Thus

$$
R^{L_{\chi}^{k}}=k R^{L_{\chi}}=k R^{L}+k \chi^{\prime}(\varrho) \partial \bar{\partial} \varrho+k \chi^{\prime \prime}(\varrho) \partial \varrho \wedge \bar{\partial} \varrho .
$$

Lemma 3.5. ([MM07, (3.5.19)]) There exists $C_{2}>0$ and $C_{3}>0$ such that, if $\chi^{\prime}(\varrho) \geq C_{3}$ on $X_{v} \backslash \bar{X}_{u}$, then

$$
\|s\|^{2} \leq \frac{C_{2}}{k}\left(\left\|\bar{\partial}_{k}^{E} s\right\|^{2}+\left\|\bar{\partial}_{k}^{E *} s\right\|^{2}\right)
$$

for $s \in B^{0, j}\left(X_{c}, L^{k} \otimes E\right)$ with $\operatorname{supp}(s) \subset X_{v} \backslash \bar{X}_{u}, j \geq q$ and $k \geq 1$, where the $L^{2}$-norm $\|\cdot\|$ is given by $\omega, h_{\chi}^{L^{k}}$ and $h^{E}$ on $X_{c}$.

Lemma 3.6. Let $\epsilon>0$ satisfying $X_{c+\epsilon} \backslash \bar{X}_{c-\epsilon}:=\{c-\epsilon<\varrho<c+\epsilon\} \Subset X_{v} \backslash \bar{X}_{u}$. Let $\phi \in \mathscr{C}_{0}^{\infty}\left(X_{v}, \mathbb{R}\right)$ with $\operatorname{supp}(\phi) \subset X_{v} \backslash \bar{X}_{u}$ such that $0 \leq \phi \leq 1$ and $\phi=1$ on $X_{c+\epsilon} \backslash \bar{X}_{c-\epsilon}$. Let $K^{\prime}:=\bar{X}_{c-\epsilon}:=\{\varrho \leq c-\epsilon\}$. Then, for any $s \in B^{0, p}\left(X_{c}, L^{k} \otimes E\right), 1 \leq p \leq n$, we have

$$
\|\phi s\|^{2} \geq\|s\|^{2}-\int_{K^{\prime}}|s| d v_{X}
$$

where the Hermitian norm $|\cdot|$ and the $L^{2}$-norm $\|\cdot\|$ are given by $\omega, h_{\chi}^{L^{k}}$ and $h^{E}$ on $X_{c}$.

Proof. For $s \in B^{0, p}\left(X_{c}, L^{k} \otimes E\right)=\operatorname{Dom}\left(\bar{\partial}_{k}^{E *}\right) \cap \Omega^{0, p}\left(\bar{X}_{c}, L^{k} \otimes E\right), \phi s \in \Omega^{0, p}\left(\bar{X}_{c}, L^{k} \otimes E\right)$ and $i_{e_{n}^{(0,1)}}(\phi s)=i_{e_{n}^{(0,1)}}(s)=0$ on $b X_{c}$ by $\phi s=s$ on the neighbourhood $X_{c+\epsilon} \backslash \bar{X}_{c-\epsilon}$ of $b X_{c}$. Thus $\phi s \in B^{0, p}\left(X_{c}, L^{k} \otimes E\right)$ with $\operatorname{supp}(\phi s) \subset X_{v} \backslash \bar{X}_{u}$,

$$
\begin{aligned}
\|\phi s\|^{2} & =\int_{X_{c}}|\phi s|^{2} d v_{X}=\int_{X_{c} \backslash \bar{X}_{u}}|\phi s|^{2} d v_{X}=\int_{\{c-\epsilon<\varrho<c\}}|\phi s|^{2} d v_{X}+\int_{u<\varrho \leq c-\epsilon}|\phi s|^{2} d v_{X} \\
& =\int_{\{c-\epsilon<\varrho<c\}}|s|^{2} d v_{X}+\int_{\{u<\varrho \leq c-\epsilon\}}|\phi s|^{2} d v_{X} \\
& \geq \int_{X_{c} \backslash \bar{X}_{c-\epsilon}}|s|^{2} d v_{X}=\|s\|^{2}-\int_{K^{\prime}}|s|^{2} d v_{X} .
\end{aligned}
$$


Lemma 3.7. Let $\phi$ be in Lemma 3.6 and let $\xi:=1-\phi$ and $C_{1}:=\sup _{x \in X_{c}}|d \xi(x)|_{g^{T^{*} X}}^{2}>0$. Then, for any $s \in B^{0, p}\left(X_{c}, L^{k} \otimes E\right), 1 \leq p \leq n$, and $k \geq 1$, we have

$$
\begin{aligned}
& \left\|\bar{\partial}_{k}^{E}(\xi s)\right\|^{2}+\left\|\bar{\partial}_{k}^{E *}(\xi s)\right\|^{2} \leq \frac{3}{2}\left(\left\|\bar{\partial}_{k}^{E} s\right\|^{2}+\left\|\bar{\partial}_{k}^{E *} s\right\|^{2}\right)+6 C_{1}\|s\|^{2}, \\
& \frac{1}{k}\left(\left\|\bar{\partial}_{k}^{E}(\phi s)\right\|^{2}+\left\|\bar{\partial}_{k}^{E *}(\phi s)\right\|^{2}\right) \leq \frac{5}{k}\left(\left\|\bar{\partial}_{k}^{E} s\right\|^{2}+\left\|\bar{\partial}_{k}^{E *} s\right\|^{2}\right)+\frac{12 C_{1}}{k}\|s\|^{2},
\end{aligned}
$$

where the $L^{2}$-norm $\|\cdot\|$ is given by $\omega, h_{\chi}^{L^{k}}$ and $h^{E}$ on $X_{c}$.

Proof. The first inequality follows from [MM07, (3.2.8)]. For simplifying notations, we use $\bar{\partial}$ and $\bar{\partial}^{*}$ instead of $\bar{\partial}_{k}^{E}$ and $\bar{\partial}_{k}^{E *}$ respectively. From $\frac{1}{2}\|\bar{\partial}(\phi s)\|^{2}-\|\bar{\partial} s\|^{2} \leq\|\bar{\partial} s-\bar{\partial}(\phi s)\|^{2}$, $\frac{1}{2}\left\|\bar{\partial}^{*}(\phi s)\right\|^{2}-\left\|\bar{\partial}^{*} s\right\|^{2} \leq\left\|\bar{\partial}^{*} s-\bar{\partial}^{*}(\phi s)\right\|^{2}$ and the first inequality, we have

$$
\frac{1}{2}\left(\|\bar{\partial}(\phi s)\|^{2}+\left\|\bar{\partial}^{*}(\phi s)\right\|^{2}\right) \leq \frac{5}{2}\left(\|\bar{\partial} s\|^{2}+\left\|\bar{\partial}^{*} s\right\|^{2}\right)+6 C_{1}\|s\|^{2},
$$

thus the second inequality follows.

Proposition 3.8. Let $X$ be a q-convex manifold of dimension $n$ with the exceptional set $K \subset X_{c}$. Then there exists a compact subset $K^{\prime} \subset X_{c}$ and $C_{0}>0$ such that for sufficiently large $k$, we have

$$
\|s\|^{2} \leq \frac{C_{0}}{k}\left(\left\|\bar{\partial}_{k}^{E} s\right\|^{2}+\left\|\bar{\partial}_{k, H}^{E *} s\right\|^{2}\right)+C_{0} \int_{K^{\prime}}|s|^{2} d v_{X}
$$

for any $s \in \operatorname{Dom}\left(\bar{\partial}_{k}^{E}\right) \cap \operatorname{Dom}\left(\bar{\partial}_{k, H}^{E *}\right) \cap L_{0, j}^{2}\left(X_{c}, L^{k} \otimes E\right)$ and $q \leq j \leq n$, where $\chi^{\prime}(\varrho) \geq C_{3}$ on $X_{v} \backslash \bar{X}_{u}$ in Lemma 3.5 and the $L^{2}$-norm is given by $\omega, h_{\chi}^{L^{k}}$ and $h^{E}$ on $X_{c}$.

Proof. We follow [MM07, Theorem 3.5.8]. Since $B^{0, j}\left(X_{c}, L^{k} \otimes E\right)$ is dense in $\operatorname{Dom}\left(\bar{\partial}_{k}^{E}\right) \cap$ $\operatorname{Dom}\left(\bar{\partial}_{k, H}^{E *}\right) \cap L_{0, j}^{2}\left(X_{c}, L^{k} \otimes E\right)$ with respect to the graph norm of $\bar{\partial}_{k}^{E}+\bar{\partial}_{k, H}^{E *}$, we only to show this inequality holds for $s \in B^{0, j}\left(X_{c}, L^{k} \otimes E\right)$ with $j \geq q$ and large $k$.

Suppose now $s \in B^{0, j}\left(X_{c}, L^{k} \otimes E\right)$. Let $\phi$ be in Lemma 3.6. Thus $\phi s \in B^{0, j}\left(X_{c}, L^{k} \otimes E\right)$ with $\operatorname{supp}(\phi s) \subset X_{v} \backslash \bar{X}_{u}$. By Lemma 3.5, there exists $C_{2}>0$ and $C_{3}>0$ such that for $j \geq q$ and $k \geq 1$, we have

$$
\|\phi s\|^{2} \leq \frac{C_{2}}{k}\left(\left\|\bar{\partial}_{k}^{E}(\phi s)\right\|^{2}+\left\|\bar{\partial}_{k}^{E *}(\phi s)\right\|^{2}\right)
$$

where $\chi^{\prime}(\varrho) \geq C_{3}$ on $X_{v} \backslash \bar{X}_{u}$ and the $L^{2}$-norm $\|\cdot\|$ is given by $\omega, h_{\chi}^{L^{k}}$ and $h^{E}$ on $X_{c}$. Next applying (3.15) and Lemma 3.6, we obtain

$$
\|s\|^{2}-\int_{K^{\prime}}|s|^{2} d v_{X} \leq \frac{5 C_{2}}{k}\left(\left\|\bar{\partial}_{k}^{E} s\right\|^{2}+\left\|\bar{\partial}_{k}^{E *} s\right\|^{2}\right)+\frac{12 C_{1} C_{2}}{k}\|s\|^{2} .
$$

For $k \geq 24 C_{1} C_{2}$, it follows that $1-\frac{12 C_{1} C_{2}}{k} \geq \frac{1}{2}$ and

$$
\|s\|^{2} \leq \frac{10 C_{2}}{k}\left(\left\|\bar{\partial}_{k}^{E} s\right\|^{2}+\left\|\bar{\partial}_{k}^{E *} s\right\|^{2}\right)+2 \int_{K^{\prime}}|s|^{2} d v_{X}
$$

The proof is comlete by choosing $C_{0}:=\max \left\{10 C_{2}, 2\right\}$ and $k \geq 24 C_{1} C_{2}$.

Thirdly, we will show that $\left(L_{\chi}, h^{L_{\chi}}\right)$ is semi-positive if $\left(L, h^{L}\right)$ is semipositive by choosing a appropriate $\chi$. Let $C_{2}>0$ and $C_{3}>0$ be in Lemma 3.5. We choose $\chi \in \mathscr{C}^{\infty}(\mathbb{R})$ such that $\chi^{\prime \prime}(t) \geq 0, \chi^{\prime}(t) \geq C_{3}$ on $(u, v)$ and $\chi(t)=0$ on $\left(-\infty, u_{0}\right)$. Therefore, 
$\chi^{\prime}(\varrho(x)) \geq C_{3}>0$ on $X_{v} \backslash \bar{X}_{u}$ and $\chi(\varrho(x))=\chi^{\prime}(\varrho(x))=0$ on $X_{u_{0}}$. Note that $K \subset X_{u_{0}}$ and $u_{0}<u<c<v$. Now we have a fixed $\chi$ which leads to the following proposition.

Proposition 3.9. Let $X$ be a q-convex manifold with $\sqrt{-1} \partial \bar{\partial} \varrho \geq 0$ on $X_{c} \backslash K$. If $\left(L, h^{L}\right)$ is semipositive on $X_{c}$, then $\left(L, h_{\chi}^{L}\right)$ is semipositive on $X_{c}$ for $\chi$ defined above.

Proof. From the above definition of $\chi$, we have $\chi^{\prime}(\varrho) \geq 0$ on $X, \chi^{\prime}(\varrho)=0$ on $K$. Since $\varrho$ is plurisubharmonic on $X_{c} \backslash K$, i.e., $\sqrt{-1} \partial \bar{\partial} \varrho \geq 0$ on $X_{c} \backslash K$, we have $\sqrt{-1} \chi^{\prime}(\varrho) \partial \bar{\partial} \varrho \geq 0$ on $X_{c}$. Since $\chi^{\prime \prime}(\varrho) \geq 0$ and $\sqrt{-1} \partial \varrho \wedge \bar{\partial} \varrho \geq 0$ on $X_{c}$, we have $\sqrt{-1} \chi^{\prime \prime}(\varrho) \partial \varrho \wedge \bar{\partial} \varrho \geq 0$ on $X_{c}$. Finally $\sqrt{-1} R^{L \chi}=\sqrt{-1} R^{L}+\sqrt{-1} \chi^{\prime}(\varrho) \partial \bar{\partial} \varrho+\sqrt{-1} \chi^{\prime \prime}(\varrho) \partial \varrho \wedge \bar{\partial} \varrho \geq 0$ on $X_{c}$.

Now we can prove the main result of this section as follows.

Proof of Theorem 3.3. By Proposition 3.9 with the fixed $\chi$, Proposition 3.8 and using Theorem 1.1 for $X_{c}$ endowed with Hermitian metric $\omega$ obtained in Lemma 3.4, there exists $C>0$ such that for any $j \geq q$ and sufficiently large $k$,

$$
\operatorname{dim} H_{(2)}^{0, j}\left(X_{c}, L^{k} \otimes E\right)=\operatorname{dim} \mathscr{H}^{0, j}\left(X_{c}, L^{k} \otimes E\right) \leq C k^{n-j}
$$

holds with respect to the metrics $\omega, h_{\chi}^{L}$ and $h^{E}$ on $X_{c}$. From [MM07, Theorem 3.5.6 (Hörmander), Theorem 3.5.7 (Andreotti-Grauert) (i), Theorem B.4.4 (The Dolbeault isomorphism)], we have for $j \geq q$,

$$
H^{j}\left(X, L^{k} \otimes E\right) \cong H^{j}\left(X_{v}, L^{k} \otimes E\right) \cong H^{0, j}\left(X_{v}, L^{k} \otimes E\right) \cong H_{(2)}^{0, j}\left(X_{c}, L^{k} \otimes E\right) .
$$

Thus the conclusion holds for sufficiently large $k$. Also we know that for any holomorphic vector bundle $F, \operatorname{dim} H^{j}(X, F)<\infty$ for $j \geq q$ by [MM07, Theorem B.4.8 (AndreottiGrauert)]. So the conclusion holds for all $k \geq 1$.

Proof of Theorem 1.4 Apply Theorem 3.3 for 1-convex manifolds.

By adapting the duality formula [HL88, 20.7 Theorem] for cohomology groups to Theorem 3.3, we have the analogue result to [Wan16, Remark 4.4] for seminegative line bundles.

Corollary 3.10. Let $X$ be a q-convex manifold of dimension $n$ with a plurisubharmonic exhaustion function $\varrho$ near the exceptional set $K$, and let $\left(L, h^{L}\right),\left(E, h^{E}\right)$ be holomorphic Hermitian line bundles on $X$. Let $\left(L, h^{L}\right)$ be seminegative on a sublevel set $X_{c}=\{x \in X$ : $\varrho(x)<c\}$ satisfying $K \subset X_{c}$. Then there exists $C>0$ such that for any $0 \leq j \leq n-q$ and $k \geq 1$, the $j$-th cohomology with compact supports

$$
\operatorname{dim}\left[H^{0, j}\left(X, L^{k} \otimes E\right)\right]_{0} \leq C k^{j} .
$$

In particular, $\operatorname{dim}\left[H^{0,0}\left(X, L^{k} \otimes E\right)\right]_{0} \leq C$.

Proof. Combine [HL88, 20.7 Theorem] and Theorem 3.3.

3.1.2. Line bundles with positivity near the exceptional set. A natural question in Theorem 3.3 is whether the hypothesis on plurisubharmonic exhaustion function is necessary. In this section, we show that such hypothesis can be replaced by the positivity assumption of line bundle near the exceptional set. 
Proof of Theorem 1.3 Let $X$ be a $q$-convex manifold of dimension $n$, let $\left(L, h^{L}\right),\left(E, h^{E}\right)$ be holomorphic Hermitian line bundles on $X$. Let $\varrho \in \mathscr{C}^{\infty}(X, \mathbb{R})$ be the exhaustion function satisfying $\sqrt{-1} \partial \bar{\partial} \varrho$ has $n-q+1$ positive eigenvalues on $X \backslash K$.

Since $\left(L, h^{L}\right)$ be semipositive on a sublevel set $X_{c}=\{x \in X: \varrho(x)<c\}$ satisfying the exceptional set $K \subset X_{c}$ and positive on $X_{c} \backslash K$, we fix real numbers $u_{0}, u, c^{\prime}$ and $v$ satisfying $u_{0}<u<c^{\prime}<v<c$ and $K \subset X_{u_{0}}$. Thus for $X_{c^{\prime}}:=\left\{x \in X: \varrho(x)<c^{\prime}\right\}$ such that $K \subset X_{c^{\prime}}$ and $L \geq 0$ on $X_{c^{\prime}}$ and $L>0$ on $X_{v} \backslash K$. For simplifying notions, we still denote $c^{\prime}$ by $c$ in this proof, that is, there exists real numbers $u_{0}, u, c$ and $v$ satisfying $u_{0}<u<c<v$ and $K \subset X_{u_{0}}$ and $L \geq 0$ on $X_{c}$ and $L>0$ on $X_{v} \backslash K$.

Firstly, we choose the metric $\omega$ on $X$ from Lemma 3.4.

Secondly, we show the fundamental estimate holds. Note $L>0$ on $X_{v} \backslash K$, by the same argument in Lemma 3.5 without modification of $h^{L}$ by $h_{\chi}^{L}$, we have that there exists $C_{2}>0$ and $k_{0}>0$ such that

$$
\|s\|^{2} \leq \frac{C_{2}}{k}\left(\left\|\bar{\partial}_{k}^{E} s\right\|^{2}+\left\|\bar{\partial}_{k}^{E *} s\right\|^{2}\right)
$$

for $s \in B^{0, j}\left(X_{c}, L^{k} \otimes E\right)$ with $\operatorname{supp}(s) \subset X_{v} \backslash \bar{X}_{u}, j \geq q$ and $k \geq k_{0}>0$, where the $L^{2}$-norm $\|\cdot\|$ is given by $\omega, h^{L^{k}}$ and $h^{E}$ on $X_{c}$. As in Lemma 3.8 without the modification of $h^{L}$, we conclude that there exist a compact subset $K^{\prime} \subset X_{c}$ (In fact, let $\epsilon>0$ such that $\{c-\epsilon<\varrho<c+\epsilon\} \Subset X_{v} \backslash \bar{X}_{u}, K^{\prime}:=\overline{\{\varrho<c-\epsilon\}}$ as in Lemma 3.6) and $C_{0}>0$ such that for sufficiently large $k$, we have

$$
\|s\|^{2} \leq \frac{C_{0}}{k}\left(\left\|\bar{\partial}_{k}^{E} s\right\|^{2}+\left\|\bar{\partial}_{k, H}^{E *} s\right\|^{2}\right)+C_{0} \int_{K^{\prime}}|s|^{2} d v_{X}
$$

for any $s \in \operatorname{Dom}\left(\bar{\partial}_{k}^{E}\right) \cap \operatorname{Dom}\left(\bar{\partial}_{k, H}^{E *}\right) \cap L_{0, j}^{2}\left(X_{c}, L^{k} \otimes E\right)$ and each $q \leq j \leq n$, where the $L^{2}$-norm is given by $\omega, h^{L^{k}}$ and $h^{E}$ on $X_{c}$.

Finally, we can apply Theorem 1.1 on $X_{c}$. Therefore, there exists $C>0$ such that for any $j \geq q$ and sufficiently large $k$,

$$
\operatorname{dim} H_{(2)}^{0, j}\left(X_{c}, L^{k} \otimes E\right) \leq C k^{n-j}
$$

holds with respect to the metrics $\omega, h^{L}$ and $h^{E}$ on $X_{c}$. As in the proof of Theorem 3.3, the conclusion holds for all $k \geq 1$.

By adapting the duality formula [HL88, 20.7 Theorem] for cohomology groups to Theorem [1.3, we have the analogue result to [Wan16, Remark 4.4] for seminegative line bundles.

Corollary 3.11. Let $X$ be a q-convex manifold of dimension $n$, and let $\left(L, h^{L}\right),\left(E, h^{E}\right)$ be holomorphic Hermitian line bundles on $X$. Let $\varrho$ be an exhaustion function of $X$ and $K$ the exceptional set. Let $\left(L, h^{L}\right)$ be seminegative on a sublevel set $X_{c}=\{x \in X: \varrho(x)<c\}$ satisfying $K \subset X_{c}$, and let $\left(L, h^{L}\right)$ be negative on $X_{c} \backslash K$. Then there exists $C>0$ such that for any $0 \leq j \leq n-q$ and $k \geq 1$, the $j$-th cohomology with compact supports

$$
\operatorname{dim}\left[H^{0, j}\left(X, L^{k} \otimes E\right)\right]_{0} \leq C k^{j} .
$$

In particular, $\operatorname{dim}\left[H^{0,0}\left(X, L^{k} \otimes E\right)\right]_{0} \leq C$.

Proof. Combining [HL88, 20.7 Theorem] and Theorem 1.3. 
Remark 3.12 (Compatibility to the vanishing theorem on $q$-convex manifolds). Let $\left(E, h^{E}\right)$ be a holomorphic vector bundle on $X$. If $\left(L, h^{L}\right)>0$ on $X_{c}$ with $K \subset X_{c}$ instead of the hypothesis $\left(L, h^{L}\right) \geq 0$ on $X_{c} \backslash K$ in Theorem 1.3 , then for $j \geq q$ and sufficiently large $k$,

$$
\operatorname{dim} H^{j}\left(X, L^{k} \otimes E\right)=0 .
$$

In particular, if $X$ is $q$-complete, then $H^{p}(X, E)=0$ for $p \geq q$ and arbitrary holomorphic vector bundle $E$. In fact, the fundamental estimates hold with $K=\emptyset$ in these cases, thus the space of harmonic forms is trivial, see [MM07, Theorem 3.5.9].

\subsection{Pseudo-convex domains.}

Theorem 3.13. Let $M \Subset X$ be a smooth strongly pseudoconvex domain in a complex manifold $X$ of dimension $n$. Let $\left(L, h^{L}\right)$ and $\left(E, h^{E}\right)$ be holomorphic Hermitian line bundles on $X$. Let $\left(L, h^{L}\right)$ be semipositive on $M$. Then there exists $C>0$ such that for any $q \geq 1$ and $k \geq 1$, we have

$$
\operatorname{dim} H^{q}\left(M, L^{k} \otimes E\right) \leq C k^{n-q} .
$$

Proof. Note a strongly pseudocovex domain is 1 -convex and applying Theorem 1.4 .

Proof of Theorem 1.5. We follow [MM07, Theorem 3.5.10]. Let $\omega$ be a Hermitian metric on $X$. Note $L>0$ around $b M$, by the same argument in Lemma 3.8 without the modification of $h^{L}$, we conclude that there exist a compact subset $K^{\prime} \subset M$ and $C_{0}>0$ such that for sufficiently large $k$,

$$
\|s\|^{2} \leq \frac{C_{0}}{k}\left(\left\|\bar{\partial}_{k}^{E} s\right\|^{2}+\left\|\bar{\partial}_{k, H}^{E *} s\right\|^{2}\right)+C_{0} \int_{K^{\prime}}|s|^{2} d v_{X}
$$

for any $s \in \operatorname{Dom}\left(\bar{\partial}_{k}^{E}\right) \cap \operatorname{Dom}\left(\bar{\partial}_{k, H}^{E *}\right) \cap L_{0, q}^{2}\left(M, L^{k} \otimes E\right)$ and each $1 \leq q \leq n$, where the $L^{2}$-norm is given by $\omega, h^{L^{k}}$ and $h^{E}$ on $M$. Finally, we apply Theorem 1.1 on $M$.

\subsection{Weakly 1-complete manifolds.}

Proof of Theorem 1.6. We follow [MM07, Theorem 3.5.12]. Let $\varphi \in \mathscr{C}^{\infty}(X, \mathbb{R})$ be an exhaustion function of $X$ such that $\sqrt{-1} \partial \bar{\partial} \varphi \geq 0$ on $X$ and $X_{c}:=\{\varphi<c\} \Subset X$ for all $c \in \mathbb{R}$. We choose a regular value $c \in \mathbb{R}$ of $\varphi$ such that $K \subset X_{c}$ by Sard's theorem. Thus $X_{c}$ is a smooth pseudoconvex domain and $L>0$ on a neighbourhood of $b X_{c}$. We apply Theorem 1.5, for any $q \geq 1$ and sufficiently large $k$

$$
\operatorname{dim} H_{(2)}^{0, q}\left(X_{c}, L^{k} \otimes E\right)=\operatorname{dim} \mathscr{H}^{0, q}\left(X_{c}, L^{k} \otimes E\right) \leq C k^{n-q} .
$$

Finally, by [Tak83, Theorem 6.2] (see [MM07, Theorem 3.5.11]) and Dolbeault isomorphism, it follows that $H^{q}\left(X, L^{k} \otimes E\right) \cong H^{q}\left(X_{c}, L^{k} \otimes E\right) \cong H^{0, q}\left(X_{c}, L^{k} \otimes E\right) \cong$ $\mathscr{H}^{0, q}\left(X_{c}, L^{k} \otimes E\right)$ for $q \geq 1$ and sufficiently large $k$.

Remark 3.14. Marinescu [Mar92] positively answered a question of Ohsawa [Ohs82, $\S 1$. Remark 2] by proving Morse inequalities on weakly 1-complete manifolds. If $L$ is $q$-positive outside a compact subset $K \subset X_{c}$, $\operatorname{dim} H^{p}\left(X_{c}, L^{k}\right)$ are at most of polynomial growth of degree $n$ with respect to $k$ for $p \geq q$. Theorem 1.6 says that if $L$ is 1-positive outside a compact subset $K \subset X_{c}$ and $L \geq 0$ on $X_{c}$ additionally, then $\operatorname{dim} H^{p}\left(X_{c}, L^{k}\right)$ are at most of polynomial growth of degree $n-p$ with respect to $k$ for $p \geq 1$.

Generally we have the following result when $\left(L, h^{L}\right)$ might be not semipositive. 
Corollary 3.15. Let $X$ be a weakly 1-complete manifold of dimension $n$. Let $\left(L, h^{L}\right)$ and $\left(E, h^{E}\right)$ be holomorphic Hermitian line bundles on $X$. Suppose there exists $f \in \mathscr{C}^{\infty}(X, \mathbb{R})$ such that $\sqrt{-1} R^{\left(L, h^{L}\right)}+\sqrt{-1} \partial \bar{\partial} f \geq 0$ on $X$, and $\sqrt{-1} R^{\left(L, h^{L}\right)}+\sqrt{-1} \partial \bar{\partial} f>0$ on $X \backslash K$ for a compact subset $K \subset X$. Then there exists $C>0$ such that for any $q \geq 1$ and sufficiently large $k$, we have

$$
\operatorname{dim} H^{q}\left(X, L^{k} \otimes E\right) \leq C k^{n-q} .
$$

Proof. Apply theorem 1.6 for the line bundle $\left(L, h^{L} e^{-f}\right)$.

Note that, by definition, there always exists a smooth function $f$ on a weakly 1-convex manifold $X$ such that $\sqrt{-1} \partial \bar{\partial} f \geq 0$, thus Corollary 3.15 implies the estimate may still hold when $\left(L, h^{L}\right)$ is not semipositive everywhere.

Remark 3.16. (Compatibility to Nakano vanishing theorem on weakly 1-convex manifolds [MM07, Theorem 3.5.15]) If $\left(L, h^{L}\right)>0$ on $X$ instead of $\left(L, h^{L}\right) \geq 0$ in Theorem 1.6, then for $q \geq 1$ and sufficiently large $k, H^{q}\left(X, L^{k} \otimes E\right)=0$, which implies the vanishing theorem on 1-convex (in particular, compact) manifold in Remark 3.12,

\subsection{Complete manifolds.}

Proof of Theorem 1.2 We follow [MM07, Theorem 3.3.5]. Since $(X, \omega)$ is complete, $\bar{\partial}_{k, H}^{E *}=\bar{\partial}_{k}^{E *}$, that is the Hilbert space adjoint and the maximal extension of the formal adjoint of $\bar{\partial}_{k}^{E}$ coincide. Let $\Lambda=i(\omega)$ be the adjoint of the operator $\omega \wedge$. with respect to the Hermitian inner product induced by $\omega, h^{L}$ and $h^{E}$. In a local orthonormal frame $\left\{\omega_{j}\right\}_{j=1}^{n}$ of $T^{(1,0)} X$ with dual frame $\left\{w^{j}\right\}_{j=1}^{n}$ of $T^{(1,0) *} X, \omega=\sqrt{-1} \sum_{j=1}^{n} \omega^{j} \wedge \bar{\omega}^{j}$ and $\Lambda=-\sqrt{-1} i_{\bar{w}_{j}} i_{w_{j}}$. Thus $\sqrt{-1} R^{\left(L, h^{L}\right)}=\sqrt{-1} \sum_{j=1}^{n} \omega^{j} \wedge \bar{\omega}^{j}$ outside $K$.

Let $F:=E \otimes K_{X}^{*}$ with Hermitian metric $h^{F}$ induced by $h^{E}$ and $\omega$. Let $\left\{e_{k}^{F}\right\}$ be a local frame of $L^{k} \otimes F$. For $s \in \Omega_{0}^{0, q}\left(X \backslash K, L^{k} \otimes E\right)=\Omega_{0}^{n, q}\left(X \backslash K, L^{k} \otimes F\right)$, we can write $s=\sum_{|J|=q} s_{J} \omega^{1} \wedge \cdots \wedge \omega^{n} \wedge \bar{\omega}^{J} \otimes e_{k}^{F}$ locally, thus

$$
\left[\sqrt{-1} R^{L}, \Lambda\right] s=\sum_{|J|=q}\left(q s_{J} \omega^{1} \wedge \cdots \wedge \omega^{n} \wedge \bar{\omega}^{J}\right) \otimes e_{k}^{F}=q s .
$$

Since $\left(X \backslash K, \sqrt{-1} R^{\left(L, h^{L}\right)}\right)$ is Kähler, we apply Nakano's inequality [MM07, (1.4.52)],

$$
\left.\left\|\bar{\partial}_{k}^{E} s\right\|^{2}+\left\|\bar{\partial}_{k}^{E *} s\right\|^{2}=\left\langle\square^{L^{k} \otimes E} s, s\right\rangle \geq k\left\langle\left[\sqrt{-1} R^{L}, \Lambda\right] s, s\right\rangle+\left[\sqrt{-1} R^{E}, \Lambda\right] s, s\right\rangle .
$$

Thus there exists $C_{E}>0$ such that, for any $1 \leq q \leq n$,

$$
\left\|\bar{\partial}_{k}^{E} s\right\|^{2}+\left\|\bar{\partial}_{k}^{E *} s\right\|^{2} \geq\left(q k-C_{E}\right)\|s\|^{2} \geq\left(k-C_{E}\right)\|s\|^{2} .
$$

Therefore, we have

$$
\|s\|^{2} \leq \frac{2}{k}\left(\left\|\bar{\partial}_{k}^{E} s\right\|^{2}+\left\|\bar{\partial}_{k}^{E *} s\right\|^{2}\right),
$$

for $s \in \Omega_{0}^{0, q}\left(X \backslash K, L^{k} \otimes E\right)$ with $1 \leq q \leq n$ and $k \geq 2 C_{E}$.

Next we follow the analogue argument in Proposition 3.8 to obtain the fundamental estimates as follows. Let $V$ and $U$ be open subsets of $X$ such that $K \subset V \Subset U \Subset X$. We choose a function $\xi \in \mathscr{C}_{0}^{\infty}(U, \mathbb{R})$ such that $0 \leq \xi \leq 1$ and $\xi \equiv 1$ on $\bar{V}$. We set $\phi:=1-\xi$, thus $\phi \in \mathscr{C}^{\infty}(X, \mathbb{R})$ satisfying $0 \leq \phi \leq 1$ and $\phi \equiv 0$ on $\bar{V}$. 
Now let $s \in \Omega_{0}^{0, q}\left(X, L^{k} \otimes E\right)$, thus $\phi s \in \Omega_{0}^{0, q}\left(X \backslash K, L^{k} \otimes E\right)$. We set $K^{\prime}:=\bar{U}$, then

$$
\|\phi s\|^{2} \geq\|s\|^{2}-\int_{K^{\prime}}|s|^{2} d v_{X}
$$

and similarly there exists a constant $C_{1}>0$ such that

$$
\frac{1}{k}\left(\left\|\bar{\partial}_{k}^{E}(\phi s)\right\|^{2}+\left\|\bar{\partial}_{k}^{E *}(\phi s)\right\|^{2}\right) \leq \frac{5}{k}\left(\left\|\bar{\partial}_{k}^{E} s\right\|^{2}+\left\|\bar{\partial}_{k}^{E *} s\right\|^{2}\right)+\frac{12 C_{1}}{k}\|s\|^{2} .
$$

By combining the above three inequalities, there exists $C_{0}>0$ such that for any $s \in$ $\Omega_{0}^{0, q}\left(X, L^{k} \otimes E\right)$ with $1 \leq q \leq n$ and $k$ large enough

$$
\|s\|^{2} \leq \frac{C_{0}}{k}\left(\left\|\bar{\partial}_{k}^{E} s\right\|^{2}+\left\|\bar{\partial}_{k}^{E *} s\right\|^{2}\right)+C_{0} \int_{K^{\prime}}|s|^{2} d v_{X} .
$$

Finally, since $\Omega_{0}^{0, \bullet}\left(X, L^{k} \otimes E\right)$ is dense in $\operatorname{Dom}\left(\bar{\partial}_{k}^{E}\right) \cap \operatorname{Dom}\left(\bar{\partial}_{k}^{E *}\right)$ in the graph-norm, for each $1 \leq q \leq n$ the fundamental estimate holds in bidegree $(0, q)$ for forms with values in $L^{k} \otimes E$ for $k$ large. So the conclusion follows from Theorem 1.1 .

\subsection{Compact manifolds and coverings revisited.}

Theorem 3.17 ([Ber02] $)$. Let $X$ be a compact complex manifold of dimension $n$. Let $\left(L, h^{L}\right)$ and $\left(E, h^{E}\right)$ be holomorphic Hermitian line bundles on $X$. Assume $\left(L, h^{L}\right)$ is semipositive on $X$. Then there exists $C>0$ such that for any $q \geq 1$ and $k \geq 1$ we have

$$
\operatorname{dim} H^{0, q}\left(X, L^{k} \otimes E\right) \leq C k^{n-q} .
$$

Note that this theorem is a special case of Theorem 1.1, 1.2, 1.3, 1.4, 1.6, 1.7 and the following Theorem 3.22 .

Corollary 3.18. Let $X$ be a compact manifold of dimension $n$, and let $\left(L, h^{L}\right),\left(E, h^{E}\right)$ be holomorphic Hermitian line bundles on $X$. Let $\left(L, h^{L}\right)$ be seminegative on $X$. Then there exists $C>0$ such that $0 \leq q \leq n-1$,

$$
\operatorname{dim} H^{0, q}\left(X, L^{k} \otimes E\right) \leq C k^{q} .
$$

In particular, $\operatorname{dim} H^{0,0}\left(X, L^{k} \otimes E\right) \leq C$.

Proof. It follows from Serre duality and Theorem 3.17 .

For the case of nef line bundles, the following observation refines the estimates in Theorem 3.17 as well as Corollary 3.18, and reflects that the magnitude $k^{n-q}$ are precise.

Lemma 3.19 ([DPS94]). Let $L$ be a nef holomorphic line bundle on a compact complex manifold $X$. Then every non-trivial section in $H^{0}\left(X, L^{-1}\right)$ has no zero at all.

Corollary 3.20. Let $X$ be a compact manifold of dimension $n$, and let $L$ be holomorphic Hermitian line bundles on $X$. Let $L$ be nef. Then, for any $k \in \mathbb{N}$,

$$
0 \leq \operatorname{dim} H^{0, n}\left(X, L^{k} \otimes K_{X}\right)=\operatorname{dim} H^{0,0}\left(X, L^{-k}\right) \leq 1
$$

Proof. Suppose there exists $k \geq 1$ such that $H^{0}\left(X, L^{-k}\right) \neq 0$, then there exists $s_{0} \in$ $H^{0}\left(X, L^{-k}\right)$ such that $s_{0}(x) \neq 0, \forall x \in X$ by Lemma 3.19, Let $s \in H^{0}\left(X, L^{-k}\right)$. It follows that $s \otimes s_{0}^{-1} \in \mathscr{O}(X)=\mathbb{C}$, thus $H^{0}\left(X, L^{-k}\right)=\mathbb{C} s_{0}$. And the case of $k=0$ is trivial. 
Remark 3.21. Let $X$ be a compact complex manifold of dimension $n$ and $\left(L, h^{L}\right)$ a holomorphic Hermitian line bundle on $X$. Let $M:=\left\{v \in L^{*}:|v|_{h^{L^{*}}}=1\right\}$. It is known that the $\bar{\partial}_{b}$ (Kohn-Rossi) cohomology $H_{b, k}^{q}(M) \cong H^{q}\left(X, L^{k}\right)$, see [MM06, Section 1.5], [HL16, (2.8)]. Thus if $L \geq 0$ on $X, \operatorname{dim} H_{b, k}^{q}(M) \leq C k^{n-q}$ for all $q \geq 1$ and $k \geq 1$.

The study of $L^{2}$ cohomology spaces on coverings of compact manifolds has also interesting applications, cf. [GHS98, Kol95]. The results are similar to the case of compact manifolds, but we have to use the reduced $L^{2}$ cohomology groups and von Neumann dimension instead of the usual dimension, see [Wan16, Theorem 1.2] or Theorem 3.22. For example, in the situation of Theorem 3.22, if the line bundle $\left(L, h^{L}\right)$ is positive, the Andreotti-Vesentini vanishing theorem [AV65] shows that $\bar{H}_{(2)}^{0, q}\left(X, L^{k} \otimes E\right) \cong$ $\mathscr{H}^{0, q}\left(X, L^{k} \otimes E\right)=0$ for $q \geq 1$ and $k$ large enough. The holomorphic Morse inequalities of Demailly [Dem85] were generalized to coverings by Chiose-Marinescu-Todor [MTC02, TCM01] (cf. also [MM07, (3.6.24)]) and yield in the conditions of Theorem 3.22 that $\operatorname{dim}_{\Gamma} \bar{H}_{(2)}^{0, q}\left(X, L^{k} \otimes E\right)=o\left(k^{n}\right)$ as $k \rightarrow \infty$ for $q \geq 1$. Hence Theorem 3.22 generalizes [Ber02] to covering manifolds and refines the estimates obtained in [MTC02, TCM01].

Theorem 3.22 ([Wan16]). Let $(X, \omega)$ be a $\Gamma$-covering manifold of dimension $n$. Let $\left(L, h^{L}\right)$ and $\left(E, h^{E}\right)$ be two $\Gamma$-invariant holomorphic Hermitian line bundles on $X$. Assume $\left(L, h^{L}\right)$ is semipositive on $X$. Then there exists $C>0$ such that for any $q \geq 1$ and $k \geq 1$ we have

$$
\operatorname{dim}_{\Gamma} \bar{H}_{(2)}^{0, q}\left(X, L^{k} \otimes E\right)=\operatorname{dim}_{\Gamma} \mathscr{H}^{0, q}\left(X, L^{k} \otimes E\right) \leq C k^{n-q} .
$$

See [Wan16, Theorem 1.2] for the complete proof. As a remark, note that for a fundamental domain $U \Subset X$ with respect to $\Gamma$, we used

$$
\operatorname{dim}_{\Gamma} \mathscr{H}^{0, q}\left(X, L^{k} \otimes E\right)=\int_{U} B_{k}^{q}(x) d v_{X}
$$

which is similar to the formula

$$
\operatorname{dim} \mathscr{H}^{0, q}\left(X, L^{k} \otimes E\right) \leq C_{0} \int_{K} B_{k}^{q}(x) d v_{X}
$$

used in the proof of Theorem 1.1.

The following estimate was firstly obtained by Morse inequalities for covering manifolds [MTC02, TCM01]. We do not assume the holomorphic Morse inequalities for coverings in our proof.

Corollary 3.23. Under the hypothesis of Theorem 3.22 if $\left(L, h^{L}\right)$ is positive at least at one point additionally, then there exists $C_{1}>0$ and $C_{2}>0$ such that for $k$ large enough

$$
C_{1} k^{n} \leq \operatorname{dim}_{\Gamma} H^{0}\left(X, L^{k} \otimes E\right) \leq C_{2} k^{n} .
$$

Proof. It follows from Riemann-Roch-Hirzenbruch formula for coverings [Ati76]

$$
\sum_{q=0}^{n}(-1)^{n-q} \operatorname{dim}_{\Gamma} \bar{H}_{(2)}^{0, q}\left(X, L^{k} \otimes E\right)=\frac{k^{n}}{n !} \int_{X / \Gamma}(-1)^{n} c_{1}\left(L / \Gamma, h^{L / \Gamma}\right)^{n}+o\left(k^{n}\right)
$$

(see also [MM07, Theorem 3.6.7]) and Theorem 3.22, 


\section{ANALYTIC SINGULAR LINE BUNDLES AND VANISHING THEOREMS}

Let $X$ be a connected compact complex manifold of dimension $n$ and $L$ a holomorphic line bundle on $X$. Let $h^{L}$ be a Hermitian metric with analytic singularities with local weight

$$
\varphi=\frac{c}{2} \log \left(\sum_{j \in J}\left|f_{j}\right|^{2}\right)+\psi
$$

where $J$ is at most countable, $c$ is a non-negative rational number, $f_{j}$ are non-zero holomorphic functions and $\psi$ is a smooth function, such that $h^{L}\left(e_{L}, e_{L}\right)=e^{-2 \varphi}$ for a local holomorphic frame $e_{L}$ of $L$. We denote by $\mathscr{J}\left(h^{L}\right):=\mathscr{J}(\varphi)$ the Nadel multiplier ideal sheaf of $h^{L}$. We define the regular part of $X$ with respect to $h^{L}$ by $R\left(h^{L}\right):=\{x \in X$ : $h^{L}$ smooth on a neighborhood of $\left.x\right\}$ and the singular part by $S\left(h^{L}\right):=X \backslash R\left(h^{L}\right)$.

4.1. Semipositive line bundles endowed metric with analytic singularities. In this section, we follow the argument of Bonavero's singular holomorphic Morse inequalities [Bon98] closely and provide three lemmas, see [MM07, 2.3.2] for details. In the end, we combine them to prove a result which is analogue to Theorem 3.17 .

Firstly, we blow up the singularities of $h^{L}$ as below, see [MM07, Lemma 2.3.19].

Lemma 4.1. There exists a proper modification $\tilde{\pi}: \widetilde{X} \longrightarrow X$ such that the local weight $\tilde{\varphi}$ of the metric $h^{\tilde{L}}=\tilde{\pi}^{*} h^{L}$ on $\tilde{L}=\tilde{\pi}^{*} L$ has the form $\varphi \circ \tilde{\pi}=\frac{c}{2} \log |g|^{2}+\tilde{\psi}$, where $g$ is holomorphic and $\tilde{\psi}$ is smooth.

Secondly, we construct a smooth hermitian metric $h^{\widehat{L}}$ on a modified line bundle $\widehat{L}$ on $\widetilde{X}$, see [MM07, Lemma 2.3.20, Lemma 2.3.21]. For a holomorphic vector bundle $F$ over $X$, we denote by $\tilde{F}:=\pi^{*} F$ the pull-back on $\widetilde{X}$.

Lemma 4.2. With the proper modification in Lemma 4.1. there exists a holomorphic line bundel $\widehat{L}$ on $\widetilde{X}$ and a smooth Hermtian metric $h^{\widehat{L}}$ satisfying the following conditions:

(1) There exists $m \in \mathbb{N} \backslash\{0\}$ such that the curvature is locally given by

$$
R^{\left(\widehat{L}, h^{\widehat{L}}\right)}=2 m \partial \bar{\partial} \tilde{\psi}
$$

(2) For any $k \in \mathbb{N} \backslash\{0\}$ and arbitrary holomorphic vector bundle $\tilde{E}$ on $\widetilde{X}$, there exists $k^{\prime} \in \mathbb{N}, m^{\prime} \in[0, m)$ with $k=m k^{\prime}+m^{\prime}$, and a holomorphic vector bundle $\widetilde{E}_{m^{\prime}}$ over $\widetilde{X}$ with $\operatorname{rank}\left(\widetilde{E}_{m^{\prime}}\right)=\operatorname{rank}(\widetilde{E})$, such that

$$
H^{q}\left(\widetilde{X}, \widetilde{L}^{k} \otimes \widetilde{E} \otimes \mathscr{J}\left(h^{\widetilde{L}^{k}}\right)\right)=H^{q}\left(\widetilde{X}, \widehat{L}^{k^{\prime}} \otimes \widetilde{E}_{m^{\prime}}\right) .
$$

Here note that there exists $C_{1}>0$ and $C_{2}>0$ such that for any integer $p \in[0, n]$ and $k$ large enough, $C_{1} k^{p} \leq k^{\prime p} \leq C_{2} k^{p}$.

Thirdly, relation to the cohomology on $X$, see [MM07, (2.3.45)].

Lemma 4.3. Let $E$ be an arbitrary holomorphic vector bundle over $X$. With the proper modification in Lemma 4.1, for all $q \geq 0$ and $k$ large enough, there exists an isomorphism

$$
H^{q}\left(X, L^{k} \otimes E \otimes \mathscr{J}\left(h^{L^{k}}\right)\right) \cong H^{q}\left(\widetilde{X}, \tilde{L}^{k} \otimes \tilde{E} \otimes K_{\tilde{X}} \otimes \widetilde{K_{X}^{*}} \otimes \mathscr{J}\left(h^{\widetilde{L}^{k}}\right)\right) .
$$

Finally we substitute these into our setting of semi-positivity of $\left(L, h^{L}\right)$ on $X$, and obtain another generalization of Theorem 3.17 . 
Proof of Theorem 1.7 Consider a local weight $\varphi$ like in (4.1) defined on an open connected subset $U \subset X$. Thus

$$
S\left(h^{L}\right) \cap U=\{x \in U: \varphi \text { not smooth at } x\}=\cap_{j \in J} Z\left(f_{j}\right),
$$

where $Z\left(f_{j}\right):=\left\{x \in U: f_{j}(x)=0\right\}$.

Let $\tilde{\pi}: \widetilde{X} \rightarrow X$ be a proper modification of Lemma 4.1, The local weight of $\left(\widetilde{L}, h^{\widetilde{L}}\right)$ on $\tilde{U}:=\tilde{\pi}^{-1}(U)$ has the form

$$
\varphi \circ \tilde{\pi}=\frac{c}{2} \log \left(\sum_{j \in J}\left|f_{j} \circ \tilde{\pi}\right|^{2}\right)+\psi \circ \tilde{\pi}=\frac{c}{2} \log |g|^{2}+\tilde{\psi},
$$

where $\tilde{\psi}$ is smooth on $\tilde{U}$. Thus $Z(g):=\{z \in \tilde{U}: g(y)=0\}$ satisfies

$$
Z(g)=\{y \in \tilde{U}: \varphi \circ \tilde{\pi} \text { not smooth at } y\}=\tilde{\pi}^{-1}\left(S\left(h^{L}\right) \cap U\right) .
$$

Since $c_{1}\left(L, h^{L}\right)=\frac{\sqrt{-1}}{2 \pi} R^{\left(L, h^{L}\right)} \geq 0$ on $X \backslash S\left(h^{L}\right), \varphi$ is smooth plurisubharmonic on $U \backslash S\left(h^{L}\right)$, i.e., $\sqrt{-1} \partial \bar{\partial} \varphi \geq 0$ on $U \backslash S\left(h^{L}\right)$. Therefore, $\varphi \circ \tilde{\pi}$ is smooth plurisubharmonic on $\tilde{\pi}^{-1}\left(U \backslash S\left(h^{L}\right)\right)=\tilde{U} \backslash Z(g)$, and since $\partial \bar{\partial} \log |g|^{2}=0$ on $\tilde{U} \backslash Z(g)$, we have on $\tilde{U} \backslash Z(g)$

$$
\sqrt{-1} \partial \bar{\partial} \tilde{\psi}=\sqrt{-1} \partial \bar{\partial}(\varphi \circ \tilde{\pi}) \geq 0 .
$$

Next we show $\sqrt{-1} \partial \bar{\partial} \tilde{\psi} \geq 0$ on $Z(g)$. In fact, suppose there exists $y_{0} \in Z(g)$ such that $\sqrt{-1} \partial \bar{\partial} \tilde{\psi}\left(y_{0}\right)$ has at least one negative eigenvalue. By the smoothness of $\tilde{\psi}$, there exists an open neighbourhood $V_{0} \subset \tilde{U}$ of $y_{0}$ satisfying $\sqrt{-1} \partial \bar{\partial} \tilde{\psi}$ has at least one negative eigenvalue on $V_{0}$. Since $Z(g)$ is nowhere dense subset in $\tilde{U}$, there exists $y_{1} \in V_{0}$ such that $y_{1} \in \tilde{U} \backslash Z(g)$. So we obtain a contradiction.

Finally, by using Lemma $4.2(1)$, we obtain $c_{1}\left(\widehat{L}, h^{\widehat{L}}\right)=\frac{\sqrt{-1}}{2 \pi} R^{\left(\widehat{L}, h^{\widehat{L}}\right)}=\frac{m}{\pi} \sqrt{-1} \partial \bar{\partial} \tilde{\psi} \geq 0$ on $\tilde{U}$, i.e., $\left(\widehat{L}, h^{\widehat{L}}\right)$ is a semipositive line bundle on $\widetilde{X}$. By (4.4), (4.3) and Theorem 3.17 applied to $\widetilde{X}$ with $\left(\widehat{L}, h^{\widehat{L}}\right)$, we have

$\operatorname{dim} H^{q}\left(X, L^{k} \otimes E \otimes \mathscr{J}\left(h^{L^{k}}\right)\right)=H^{q}\left(\widetilde{X}, \widehat{L}^{k^{\prime}} \otimes\left(\widetilde{E} \otimes K_{\tilde{X}} \otimes \widetilde{K_{X}^{*}}\right)_{m^{\prime}}\right) \leq C k^{\prime n-q} \leq C C_{2} k^{n-q}$.

Since a positive current $c_{1}\left(L, h^{L}\right)$ on $X$ is semipositive on $R\left(h^{L}\right)$, the last assertion follows.

In analogy to the covering manifolds case, we have the following estimate of the space of holomorphic sections obtained firstly by Bonavero, see [Bou02] and [MM07, Corollary 2.3.46]. We remark that we do not use Bonavero's holomorphic Morse inequalities in our proof.

Corollary 4.4. Under the hypothesis of Theorem 1.7 if $\left(L, h^{L}\right)$ is positive at least at one point additionally, then for $k$ large enough

$$
C_{1} k^{n} \leq \operatorname{dim} H^{0}\left(X, L^{k} \otimes E \otimes \mathscr{J}\left(h^{L^{k}}\right)\right) \leq C_{2} k^{n} .
$$

In particular, if $X$ is additionally Kähler, then $X$ is projective.

Proof. It follows from Theorem 1.7 and asymptotic Riemann-Roch-Hirzenbruch formula for $h^{L}$ with analytic singularities (see [MM07, (2.3.45), (2.3.31), (1.7.1)])

$$
\sum_{q=0}^{n}(-1)^{n-q} \operatorname{dim} H^{q}\left(X, L^{k} \otimes E \otimes \mathscr{J}\left(h^{L^{k}}\right)\right)=\frac{k^{n}}{n !} \int_{R\left(h^{L}\right)}(-1)^{n} c_{1}\left(L, h^{L}\right)^{n}+o\left(k^{n}\right),
$$


where $R\left(h^{L}\right):=\left\{x \in X: h^{L}\right.$ smooth on a neighborhood of $\left.x\right\}$ is the regular part of $X$ with respect to $h^{L}$. Thus $L$ is big, $X$ is Moishezon. So $X$ is projective when it is Kähler.

4.2. Vanishing theorems for singular line bundles. In this section, let $X$ be a compact complex manifold of dimension $n$, let $E$ and $L$ be holomorphic vector bundles over $X$ with $\operatorname{rank}(L)=1$. The key observation in the following Theorem 1.9 and 4.7 is that the curvature conditions of line bundles are preserved under the proper modifications (in Lemma 4.1) as in the proof of Theorem 1.7, therefore, the proof of Theorem 1.9 and 4.7 are analogue to the argument in Theorem 1.7.

The vanishing theorems for partially positive line bundles [Dem, Ch.VII.(5.1)]) entails that for arbitrary smooth Hermitian metric $h^{L}$ on $L$, if $c_{1}\left(L, h^{L}\right)$ has at least $n-s+1$ positive eigenvalues on $X$, then

$$
H^{q}\left(X, E \otimes L^{k}\right)=0
$$

for $q \geq s$ and sufficiently large $k$. Similarly we obtain the analytic singular version of (4.11) as follows.

Proof of Theorem 1.9 Consider a local weight $\varphi$ like in (4.1) defined on an open connected subset $U \subset X$. Thus

$$
S\left(h^{L}\right) \cap U=\{x \in U: \varphi \text { not smooth at } x\}=\cap_{j \in J} Z\left(f_{j}\right),
$$

where $Z\left(f_{j}\right):=\left\{x \in U: f_{j}(x)=0\right\}$.

Let $\tilde{\pi}: \widetilde{X} \rightarrow X$ be a proper modification of Lemma 4.1. Note $\tilde{\pi}: \widetilde{X} \backslash \tilde{B} \cong X \backslash B$ is biholomorphic and $B \subset X$ and $\tilde{B} \subset \widetilde{X}$ are nowhere dense (closed) analytic subsets. The local weight of $\left(\tilde{L}, h^{\tilde{L}}\right)$ on $\tilde{U}:=\tilde{\pi}^{-1}(U)$ has the form

$$
\varphi \circ \tilde{\pi}=\frac{c}{2} \log \left(\sum_{j \in J}\left|f_{j} \circ \tilde{\pi}\right|^{2}\right)+\psi \circ \tilde{\pi}=\frac{c}{2} \log |g|^{2}+\tilde{\psi},
$$

where $\tilde{\psi}$ is smooth on $\tilde{U}$. Thus $Z(g):=\{z \in \tilde{U}: g(y)=0\}$ satisfies

$$
Z(g)=\{y \in \tilde{U}: \varphi \circ \tilde{\pi} \text { not smooth at } y\}=\tilde{\pi}^{-1}\left(S\left(h^{L}\right) \cap U\right) .
$$

Firstly, we show the number of positive eigenvalues was preserved by $\tilde{\pi}$ on $\widetilde{X}$.

Since $\sqrt{-1} \partial \bar{\partial} \varphi$ has at least $n-s+1$ positive eigenvalues on $U \backslash S$, and

$$
\sqrt{-1} \partial \bar{\partial} \tilde{\psi}=\sqrt{-1} \partial \bar{\partial}(\varphi \circ \tilde{\pi}) \quad \text { on } \tilde{U} \backslash Z(g),
$$

we see $\sqrt{-1} \partial \bar{\partial} \tilde{\psi}$ has at least $n-s+1$ positive eigenvalues on $\tilde{U} \backslash(Z(g) \cup \tilde{B})$.

Next we show $\sqrt{-1} \partial \bar{\partial} \tilde{\psi}$ has at least $n-s+1$ positive eigenvalues on $\tilde{U} \cap(Z(g) \cup \tilde{B})$, thus on $\tilde{U}$. In fact, suppose $\sqrt{-1} \partial \bar{\partial} \tilde{\psi}$ has no more than $n-s$ positive eigenvalues at $x_{0} \in \tilde{U} \cap(Z(g) \cup \tilde{B})$. By the smoothness of $\tilde{\psi}$, there exists a neighbourhood $V_{0} \subset \tilde{U}$ of $x_{0}$ such that $\sqrt{-1} \partial \bar{\partial} \tilde{\psi}$ has no more than $n-s$ positive eigenvalues on $V_{0}$. Since $\tilde{B}$ is nowhere dense, there exists $y_{0} \in V_{0} \backslash \tilde{B}$. If $y_{0}$ is not in $Z(g), y_{0} \in V_{0} \backslash(\tilde{B} \cup Z(g)) \subset \tilde{U} \backslash(\tilde{B} \cup Z(g))$ which leads to a contradiction. If $y_{0} \in Z(g), y_{0} \in\left(V_{0} \cap Z(g)\right) \backslash \tilde{B}$. There exists a neighbourhood $W_{0} \subset V_{0} \backslash \tilde{B}$ of $y_{0}$ such that $\sqrt{-1} \partial \bar{\partial} \tilde{\psi}$ has no more than $n-s$ positive eigenvalues on $W_{0}$. But $W_{0}$ is not a subset of the nowhere dense set $Z(g)$, thus there exists a point $z_{0} \in W_{0} \backslash Z(g) \subset V_{0} \backslash(\tilde{B} \cup Z(g)) \subset \tilde{U} \backslash(\tilde{B} \cup Z(g))$, and $\left.\sqrt{-1} \partial \bar{\partial} \tilde{\psi}\right|_{z_{0}}$ has no more than $n-s$ positive eigenvalues. So it is a contradiction. 
By using Lemma $4.2(1), c_{1}\left(\widehat{L}, h^{\widehat{L}}\right)=\frac{\sqrt{-1}}{2 \pi} R^{\left(\widehat{L}, h^{\widehat{L}}\right)}=\frac{m}{\pi} \sqrt{-1} \partial \bar{\partial} \tilde{\psi}$ on $\tilde{U},\left(\widehat{L}, h^{\widehat{L}}\right)$ has at least $n-s+1$ positive eigenvalues on $\widetilde{X}$. By (4.4), (4.3) and (4.11) applied to $\widetilde{X}$ with $\left(\widehat{L}, h^{\widehat{L}}\right)$, we have for $q \geq s$ and sufficiently large $k$,

$$
\operatorname{dim} H^{q}\left(X, L^{k} \otimes E \otimes \mathscr{J}\left(h^{L^{k}}\right)\right)=H^{q}\left(\widetilde{X}, \widehat{L}^{k^{\prime}} \otimes\left(\tilde{E} \otimes K_{\tilde{X}} \otimes \widetilde{K_{X}^{*}}\right)_{m^{\prime}}\right)=0 .
$$

$X(t):=\left\{x \in R\left(h^{L}\right): c_{1}\left(L, h^{L}\right)_{x}\right.$ is non-degenerated with exactly $t$ negative eigenvalues $\}$ and $X(\leq s-1):=\cup_{0 \leq t \leq s-1} X(t)$. Finally, the proof is complete by (4.10).

Corollary 4.5. Let $X$ be a compact manifold of dimension $n$, let $L$ and $E$ be holomorphic line bundles on $X$. Let $h^{L}$ be the Hermitian metric on $L$ with analytic singularities as in (4.1). Let $0 \leq t \leq n$. Assume $c_{1}\left(L, h^{L}\right) \geq 0$ on $R\left(h^{L}\right)$ and $c_{1}\left(L, h^{L}\right)$ has at least $n-t$ positive eigenvalues on $R\left(h^{L}\right)$.

Then, as $k \rightarrow \infty$, we have

(4.17) $\operatorname{dim} H^{q}\left(X, E \otimes L^{k} \otimes \mathscr{J}\left(h^{L^{k}}\right)\right)= \begin{cases}\frac{k^{n}}{n !} \int_{X(0)} c_{1}\left(L, h^{L}\right)^{n}+o\left(k^{n}\right), & \text { for } q=0, \\ \mathcal{O}\left(k^{n-q}\right), & \text { for } 1 \leq q \leq t, \\ 0, & \text { for } q>t .\end{cases}$

Proof. The third equality is from Theorem 1.9, the second equality is from Theorem 1.7 and the first is given by combining the second equality and (1.14).

Remark 4.6. As a remark on Corollary 4.5, if $t=0,\left(L, h^{L}\right)$ is positive on $R\left(h^{L}\right)$; if $t=n$, $\left(L, h^{L}\right)$ is semipositive on $R\left(h^{L}\right)$. The smooth version (i.e., $h^{L}$ is smooth everywhere) of Corollary 4.5] are given by [Dem85], [Ber02] and [Dem, Ch.VII.(5.1)] respectively.

By [Rie71, Appendix, Theorem 6 and Theorem 3 (ii)], for a compact complex manifold $X$ and a holomorphic Hermitian line bundle $\left(L, h^{L}\right)$ on $X$, if $X$ is Moishezon and $c_{1}\left(L, h^{L}\right) \geq 0$ on $X$ and $c_{1}\left(L, h^{L}\right)>0$ at least at one point, then $H^{q}\left(X, K_{X} \otimes L\right)=0$ for all $q \geq 1$. Furthermore, Siu's resolution [Siu85, Theorem 1 (see Page 175)]) of GrauertRiemenschneider conjecture shows that the Moishezon assumption is unnecessary, see also [Dem, Ch.VII. (3.5) Theorem]. Thus, Siu's vanishing theorem entails that, for arbitrary smooth Hermitian metric $h^{L}$ on $L$, if $c_{1}\left(L, h^{L}\right) \geq 0$ on $X$ and $c_{1}\left(L, h^{L}\right)>0$ at least at one point, then

$$
H^{q}\left(X, K_{X} \otimes L\right)=0
$$

for $q \geq 1$. Similarly, the analytic singular version of (4.18) is as follows.

Theorem 4.7 (Theorem 1.10). Let $X$ be a compact manifold of dimension $n$, let $L$ be a holomorphic line bundle. Let $h^{L}$ be the Hermitian metric on $L$ with analytic singularities as in (4.1). If $c_{1}\left(L, h^{L}\right) \geq 0$ on $R\left(h^{L}\right)$ and $c_{1}\left(L, h^{L}\right)>0$ at least at one point in $R\left(h^{L}\right)$, then

$$
H^{q}\left(X, K_{X} \otimes L^{k m} \otimes \mathscr{J}\left(h^{L^{k m}}\right)\right)=0
$$

for $q \geq 1, k \geq 1$ and $m \in \mathbb{N} \backslash\{0\}$ satisfying $m c \in \mathbb{N}$ with non-negative rational number $c$ in (4.1).

Proof. Consider a local weight $\varphi$ like in (4.1) defined on an open connected subset $U \subset$ $X$. Thus

$$
S\left(h^{L}\right) \cap U=\{x \in U: \varphi \text { not smooth at } x\}=\cap_{j \in J} Z\left(f_{j}\right),
$$


where $Z\left(f_{j}\right):=\left\{x \in U: f_{j}(x)=0\right\}$.

Let $\tilde{\pi}: \widetilde{X} \rightarrow X$ be a proper modification of Lemma 4.1. Note $\tilde{\pi}: \widetilde{X} \backslash \tilde{B} \cong X \backslash B$ is biholomorphic and $B \subset X$ and $\tilde{B} \subset \widetilde{X}$ are nowhere dense (closed) analytic subsets. The local weight of $\left(\tilde{L}, h^{\widetilde{L}}\right)$ on $\tilde{U}:=\tilde{\pi}^{-1}(U)$ has the form

$$
\varphi \circ \tilde{\pi}=\frac{c}{2} \log \left(\sum_{j \in J}\left|f_{j} \circ \tilde{\pi}\right|^{2}\right)+\psi \circ \tilde{\pi}=\frac{c}{2} \log |g|^{2}+\tilde{\psi}
$$

where $\tilde{\psi}$ is smooth on $\tilde{U}$. Thus $Z(g):=\{z \in \tilde{U}: g(y)=0\}$ satisfies

$$
Z(g)=\{y \in \tilde{U}: \varphi \circ \tilde{\pi} \text { not smooth at } y\}=\tilde{\pi}^{-1}\left(S\left(h^{L}\right) \cap U\right) .
$$

Firstly, we have known that the semipositivity was preserved by $\tilde{\pi}$ on $\widetilde{X}$ in the proof of Theorem 1.7, i.e., $\sqrt{-1} \partial \bar{\partial} \tilde{\psi} \geq 0$ on $\tilde{U}$.

Secondly, we show the positivity at some point was preserved by $\tilde{\pi}$. Let $U \subset X$ be a neighbourhood of this point such that $\left(L, h^{L}\right)$ is positive on $U \backslash S\left(h^{L}\right)$. Since $\sqrt{-1} \partial \bar{\partial} \varphi$ is positive on $U \backslash S\left(h^{L}\right)$, and on $\tilde{U} \backslash Z(g)$

$$
\sqrt{-1} \partial \bar{\partial} \tilde{\psi}=\sqrt{-1} \partial \bar{\partial}(\varphi \circ \tilde{\pi})
$$

$\sqrt{-1} \partial \bar{\partial} \tilde{\psi}$ is positive on $\tilde{U} \backslash(Z(g) \cup \tilde{B})$ which is not empty, since the nowhere dense of $\tilde{B}$ and the analytic subset $Z(g) \subset \widetilde{U}$.

Finally, Lemma 4.2 $(1) c_{1}\left(\widehat{L}, h^{\widehat{L}}\right)=\frac{\sqrt{-1}}{2 \pi} R^{\left(\widehat{L}, h^{\widehat{L}}\right)}=\frac{m}{\pi} \sqrt{-1} \partial \bar{\partial} \tilde{\psi}$ on $\tilde{U}$ implies $\left(\widehat{L}, h^{\widehat{L}}\right)$ is semipositve on $\widetilde{X}$ and positive at least at one point. By (4.4), (4.3) and (4.18) applied to $\widetilde{X}$ with $\left(\widehat{L}, h^{\widehat{L}}\right)$, we have for $q \geq 1$ and $k \geq 1$,

$\operatorname{dim} H^{q}\left(X, K_{X} \otimes L^{k m} \otimes \mathscr{J}\left(h^{L^{k m}}\right)\right)=H^{q}\left(\widetilde{X}, K_{\tilde{X}} \otimes \widetilde{L}^{k m} \otimes \mathscr{J}\left(h^{\widetilde{L}^{k m}}\right)\right)=H^{q}\left(\widetilde{X}, K_{\tilde{X}} \otimes \hat{L}^{k}\right)=0$, where $m \in \mathbb{N} \backslash\{0\}$ is arbitrary number satisfying $m c \in \mathbb{N}$ (see [MM07, (2.3.27), (2.3.32)]).

Corollary 4.8. Under the hypothesis of Theorem 4.7. if $c \in \mathbb{N}$ in (4.1) additionally, then

$$
H^{q}\left(X, K_{X} \otimes L \otimes \mathscr{J}\left(h^{L}\right)\right)=0
$$

for $q \geq 1$. In particular, if $c \in \mathbb{N}$ in (4.1) and $L=K_{X}^{*}$ additionally, then

$$
H^{q}\left(X, \mathscr{J}\left(h^{K_{X}^{*}}\right)\right)=0
$$

for $q \geq 1$, and the Euler characteristic

$$
\chi\left(X, \mathscr{J}\left(h^{K_{X}^{*}}\right)\right)=\operatorname{dim} H^{0}\left(X, \mathscr{J}\left(h^{K_{X}^{*}}\right)\right) \leq 1 .
$$

Proof. Take $m=k=1$ in Theorem 4.7 .

\section{ACKNOWLEDGEMENTS}

The author thanks Professor Xianzhe Dai, Professor George Marinescu for helpful discussion and enlightened comments. This work is partially supported by Albert's Researcher Reunion Grant of University of Cologne. 


\section{REFERENCES}

[AG62] Aldo Andreotti and Hans Grauert. Théorème de finitude pour la cohomologie des espaces complexes. Bull. Soc. Math. France, 90:193-259, 1962.

[Ati76] M. F. Atiyah. Elliptic operators, discrete groups and von Neumann algebras. pages 43-72. Astérisque, No. 32-33, 1976.

[AV65] Aldo Andreotti and Edoardo Vesentini. Carleman estimates for the Laplace-Beltrami equation on complex manifolds. Inst. Hautes Études Sci. Publ. Math., (25):81-130, 1965; Erratum: (27):153$155,1965$.

[Ber02] Bo Berndtsson. An eigenvalue estimate for the $\bar{\partial}$-Laplacian. J. Differential Geom., 60(2):295313, 2002.

[Bon98] Laurent Bonavero. Inégalités de morse holomorphes singulières. J. Geom. Anal., 8(3):409-425, 1998.

[Bou02] Sébastien Boucksom. On the volume of a line bundle. Internat. J. Math., 13(10):1043-1063, 2002.

[CM15] Dan Coman and George Marinescu. Equidistribution results for singular metrics on line bundles. Ann. Sci. Éc. Norm. Supér. (4), 48(3):497-536, 2015.

[Dem] Jean-Pierre Demailly. Complex analytic and differential geometry. Available at http://www-fourier.ujf-grenoble.fr/ demailly/manuscripts/agbook.pdf.

[Dem85] Jean-Pierre Demailly. Champs magnétiques et inégalités de Morse pour la $d^{\prime \prime}$-cohomologie. Ann. Inst. Fourier (Grenoble), 35(4):189-229, 1985.

[Dem92] Jean-Pierre Demailly. Singular Hermitian metrics on positive line bundles. In Complex algebraic varieties (Bayreuth, 1990), volume 1507 of Lecture Notes in Math., pages 87-104. Springer, Berlin, 1992.

[DPS94] Jean-Pierre Demailly, Thomas Peternell, and Michael Schneider. Compact complex manifolds with numerically effective tangent bundles. J. Algebraic Geom., 3(2):295-345, 1994.

[GHS98] Mikhael Gromov, Gennadi Markovič Henkin, and Mikhail A. Shubin. Holomorphic $L^{2}$ functions on coverings of pseudoconvex manifolds. Geom. Funct. Anal., 8(3):552-585, 1998.

[GR70] Hans Grauert and Oswald Riemenschneider. Verschwindungssätze für analytische Kohomologiegruppen auf komplexen Räumen. Invent. Math., 11:263-292, 1970.

[HL88] Gennadi M. Henkin and Jürgen Leiterer. Andreotti-Grauert theory by integral formulas, volume 74 of Progress in Mathematics. Birkhäuser Boston, Inc., Boston, MA, 1988.

[HL16] Chin-Yu Hsiao and Xiaoshan Li. Morse inequalities for Fourier components of Kohn-Rossi cohomology of CR manifolds with $S^{1}$-action. Math. Z., 284(1-2):441-468, 2016.

[HM14] Chin-Yu Hsiao and George Marinescu. Asymptotics of spectral function of lower energy forms and Bergman kernel of semi-positive and big line bundles. Comm. Anal. Geom., 22(1):1-108, 2014.

[Kol95] János Kollár. Shafarevich maps and automorphic forms. M. B. Porter Lectures. Princeton University Press, Princeton, NJ, 1995.

[Mar92] George Marinescu. Morse inequalities for $q$-positive line bundles over weakly 1-complete manifolds. C. R. Acad. Sci. Paris Sér. I Math., 315(8):895-899, 1992.

[MM06] Xiaonan Ma and George Marinescu. The first coefficients of the asymptotic expansion of the Bergman kernel of the $\mathrm{Spin}^{c}$ Dirac operator. Internat. J. Math., 17(6):737-759, 2006.

[MM07] Xiaonan Ma and George Marinescu. Holomorphic Morse inequalities and Bergman kernels, volume 254 of Progress in Mathematics. Birkhäuser Verlag, Basel, 2007.

[MTC02] George Marinescu, Radu Todor, and Ionuţ Chiose. $L^{2}$ holomorphic sections of bundles over weakly pseudoconvex coverings. Geom. Dedicata, 91:23-43, 2002.

[Nad90] Alan Michael Nadel. Multiplier ideal sheaves and Kähler-Einstein metrics of positive scalar curvature. Ann. of Math. (2), 132(3):549-596, 1990.

[Ohs82] Takeo Ohsawa. Isomorphism theorems for cohomology groups of weakly 1-complete manifolds. Publ. Res. Inst. Math. Sci., 18(1):191-232, 1982. 
[Rie71] Oswald Riemenschneider. Characterizing Moišezon spaces by almost positive coherent analytic sheaves. Math. Z., 123:263-284, 1971.

[Siu84] Yum Tong Siu. A vanishing theorem for semipositive line bundles over non-Kähler manifolds. $J$. Differential Geom., 19(2):431-452, 1984.

[Siu85] Yum Tong Siu. Some recent results in complex manifold theory related to vanishing theorems for the semipositive case. In Workshop Bonn 1984 (Bonn, 1984), volume 1111 of Lecture Notes in Math., pages 169-192. Springer, Berlin, 1985.

[Tak83] Kenshō Takegoshi. Global regularity and spectra of Laplace-Beltrami operators on pseudoconvex domains. Publ. Res. Inst. Math. Sci., 19(1):275-304, 1983.

[TCM01] Radu Todor, Ionuţ Chiose, and George Marinescu. Morse inequalities for covering manifolds. Nagoya Math. J., 163:145-165, 2001.

[Wan16] Huan Wang. On the growth of von Neumann dimension of harmonic spaces of semipositive line bundles over covering manifolds. Internat. J. Math., 27(11):1650093, 14, 2016.

School of Mathematical Sciences, Shanghai Key laboratory of PMMP, East China Normal University, Dong ChuAn RoAd 500, Shanghai 200241, P. R. China

E-mail address: huanwang2016@hotmail.com, hwang@math.ecnu.edu.cn 